\title{
Soluble fms-like tyrosine kinase 1 promotes angiotensin II sensitivity in preeclampsia
}

\author{
Suzanne D. Burke, ${ }^{1,2,3}$ Zsuzsanna K. Zsengellér, ${ }^{1,4}$ Eliyahu V. Khankin, ${ }^{1,2,3}$ Agnes S. Lo, ${ }^{1,2,3}$ Augustine Rajakumar, ${ }^{1,2,3}$ \\ Jennifer J. DuPont, ${ }^{5}$ Amy McCurley, ${ }^{5}$ Mary E. Moss, ${ }^{5,6}$ Dongsheng Zhang, ${ }^{1,2,3}$ Christopher D. Clark, ${ }^{1,2,3}$ Alice Wang, ${ }^{1,2}$ \\ Ellen W. Seely, ${ }^{3,7}$ Peter M. Kang,,$^{1,2,3}$ Isaac E. Stillman, ${ }^{3,4}$ Iris Z. Jaffe, ${ }^{5,8}$ and S. Ananth Karumanchi ${ }^{1,2,3}$ \\ 'Department of Medicine and Department of Obstetrics and Gynecology, and ${ }^{2}$ Center for Vascular Biology Research, Beth Israel Deaconess Medical Center, Boston, Massachusetts, USA. ${ }^{3}$ Harvard Medical

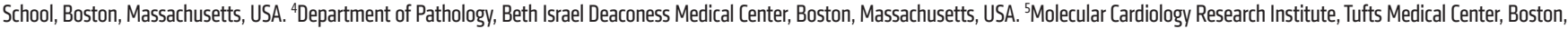 \\ Massachusetts, USA. ${ }^{6}$ Sackler School of Biomedical Graduate Studies, Tufts University School of Medicine, Boston, Massachusetts, USA. ${ }^{7}$ Division of Endocrinology, Diabetes and Hypertension, \\ Department of Medicine, Brigham and Women's Hospital, Boston, Massachusetts, USA. ${ }^{8}$ Division of Cardiology, Tufts Medical Center, Boston, Massachusetts, USA
}

\begin{abstract}
Preeclampsia is a hypertensive disorder of pregnancy in which patients develop profound sensitivity to vasopressors, such as angiotensin II, and is associated with substantial morbidity for the mother and fetus. Enhanced vasoconstrictor sensitivity and elevations in soluble fms-like tyrosine kinase 1 (sFLT1), a circulating antiangiogenic protein, precede clinical signs and symptoms of preeclampsia. Here, we report that overexpression of sFIt1 in pregnant mice induced angiotensin II sensitivity and hypertension by impairing endothelial nitric oxide synthase (eNOS) phosphorylation and promoting oxidative stress in the vasculature. Administration of the NOS inhibitor L-NAME to pregnant mice recapitulated the angiotensin sensitivity and oxidative stress observed with sFIt1 overexpression. Sildenafil, an FDA-approved phosphodiesterase 5 inhibitor that enhances NO signaling, reversed sFlt1-induced hypertension and angiotensin II sensitivity in the preeclampsia mouse model. Sildenafil treatment also improved uterine blood flow, decreased uterine vascular resistance, and improved fetal weights in comparison with untreated sFlt1-expressing mice. Finally, sFLT1 protein expression inversely correlated with reductions in eNOS phosphorylation in placental tissue of human preeclampsia patients. These data support the concept that endothelial dysfunction due to high circulating sFLT1 may be the primary event leading to enhanced vasoconstrictor sensitivity that is characteristic of preeclampsia and suggest that targeting sFLT1-induced pathways may be an avenue for treating preeclampsia and improving fetal outcomes.
\end{abstract}

\section{Introduction}

Patients with preeclampsia, a devastating hypertensive complication that occurs after the 20th week of gestation, exhibit a significantly altered angiogenic and hemodynamic profile compared with normal pregnancy $(1,2)$. In a seminal publication by Gant and colleagues, pregnancy was determined to be a state of relative insensitivity to direct infusion of the pressor hormone angiotensin II (Ang II), a physiological adaptation that contributes to low systemic vascular resistance (3). Conversely, pregnant women who later developed preeclampsia remained sensitive to the pressor effects of Ang II, requiring increasingly smaller doses to achieve the same rise in blood pressure. However, this phenomenon is not specific to Ang II, as sensitivity to other pressors, including catecholamines, has also been reported in preeclampsia $(4,5)$. Ang II sensitivity has since been well described to occur prior to the clinical signs and symptoms of preeclampsia $(3,6)$, yet the mechanism remains unknown, thereby preventing therapeutic intervention. Several

Conflict of interest: S.A. Karumanchi is a coinventor on patents related to preeclampsia biomarkers that have been out-licensed to multiple companies. S.A. Karumanchi has financial interest in Aggamin LLC and reports serving as a consultant to Siemens Diagnostics, Roche, and Thermo Fisher.

Submitted: July 24, 2015; Accepted: April 7, 2016.

Reference information: J Clin Invest. 2016;126(7):2561-2574. doi:10.1172/JCI83918. hypotheses have been proposed, including agonistic autoantibodies to the Ang II type 1 receptor (AT1R) $(7,8)$, AT1R and bradykinin $\mathrm{B}_{2}$ heterodimerization $(9,10)$, regulation of $\mathrm{G}$ protein signaling $(11)$, and activation of uteroplacental renin-angiotensin system (RAS) $(12,13)$. These postulates have yielded critical pathophysiological data; however, as yet no mechanism for the generalized sensitivity to vasopressors in preeclampsia has been elucidated.

Epidemiological and experimental studies support a pathophysiological role for excess circulating soluble fms-like tyrosine kinase 1 (sFLT1) in preeclampsia (1). sFLT1 is a soluble variant of VEGF receptor 1, which acts as an antiangiogenic trap for VEGF and placental growth factor in tissues and in circulation (14). Circulating levels of sFLT1 are elevated not only during clinical disease, but also 4-6 weeks before the onset of clinical signs and symptoms of preeclampsia (15-17). The observation of a temporal relationship between high circulating sFLT1 and Ang II sensitivity led us to hypothesize that sFLT1 may directly influence the increased sensitivity to Ang II in preeclampsia. We therefore sought to determine, in normal and $s F l t 1$-overexpressing mice, the mechanism of Ang II sensitivity during pregnancy.

\section{Results}

Ang II response in normal pregnant and preeclamptic mice mimics human physiology. To confirm that mice exhibit Ang II insensitiv- 
ity during gestation as observed in women, we used in vivo and ex vivo approaches. Chronic infusion of a pressor dose of Ang II (0.43 mg $/ \mathrm{kg} / \mathrm{d}$ via s.c. osmotic minipump) to nonpregnant mice induced a predictable rise in blood pressure of more than 40 mmHg (Figure 1, A and B; $P<0.001$ ). The same dose of Ang II administered to pregnant mice at gestational day (gd) 12 elicited a maximum increase in blood pressure of $22 \mathrm{mmHg}$. This increase was significant compared with mid-gestation nadir (the normal decline in blood pressure observed at gd8-10; Figure 1, A and B; $P<0.05)$, but not with early or nonpregnant values. The change $(\Delta)$ in blood pressure after chronic Ang II infusion was significantly less in pregnant compared with nonpregnant mice (Figure 1B; $P<0.001$ ), confirming resistance to Ang II in normal pregnancy. We used the well-characterized $s F l t 1$ overexpression model to induce a preeclampsia-like condition in pregnant mice that reproduces the human disorder (18-20). Hemodynamic parameters were similar between sFlt1-treated and CMV-null-treated pregnant mice until gd12 (4 days after injection), when mean arterial pressure increased significantly in sFlt1-overexpressing mice (Figure $1, \mathrm{C}$ and $\mathrm{D} ; P<0.01)$. We also confirmed preeclamptic phenotype by postmortem examination of kidneys, which revealed characteristic glomerular endotheliosis-like lesions in sFlt1-overexpressing mice (Supplemental Figure 1A; supplemental material available online with this article; doi:10.1172/JCI83918DS1).

The blood pressure response in the $s F l t 1$-induced preeclampsia model was next examined in response to acute Ang II bolus injection in conscious, unrestrained mice while they were nonpregnant and then at predetermined gestational dates. Acute i.v. injection of Ang II into nonpregnant mice caused a reproducible increase in blood pressure $(30 \pm 5.9 \mathrm{mmHg})$ within 5 minutes, which normalized to preinjection levels within 25 minutes $(n=8$; Supplemental Figure 1B). The pressor response to Ang II in normal pregnant mice became blunted at gd10, and then was refractory at gd14 $(-3.6 \pm 1.8 \mathrm{mmHg}$ averaged over 15 minutes after injection; Figure 1E; $P<0.05)$. Comparable reductions were observed in heart rate at gd14 $(-73.6 \pm 13.1 \mathrm{bpm}$ compared with nonpregnant; Supplemental Figure 1, C and D; $P<0.05)$. Late-gestation response to Ang II remained blunted with return to normal response postpartum. In pregnant mice treated with $s F l t 1$, response to acute bolus injection of Ang II induced a hemodynamic response similar to that observed in the nonpregnant state (Figure 1E). These data demonstrate that sensitivity to acute infusions of Ang II is blunted in normal mid- to late murine pregnancy, while preeclamptic mice remain sensitive to acute Ang II exposure throughout pregnancy.

Consistent with the acute data, chronic infusion of Ang II caused a more rapid increase in blood pressure in sFlt1-treated preeclamptic mice (Figure 1F). Chronic Ang II increased blood pressure in both CMV-null (control pregnant) and sFlt1 pregnant mice, although the magnitude of change was significant in only the sFlt1 mice (Figure 1G; $P<0.01$ ). We confirmed that plasma sFLT1 levels at gd17 were significantly elevated in sFlt1-injected mice compared with CMV-null $(P<0.001)$, and that chronic Ang II infusion did not alter plasma sFLT1 levels (Supplemental Figure $1 \mathrm{E})$. These data indicate that increased circulating sFLT1 may contribute to the maintenance of responsiveness to vasoconstrictors observed in preeclamptic women. To further explore this idea, we isolated second-order mesenteric resistance vessels from gd17
CMV-null or sFlt1-treated mice for ex vivo contraction studies (Figure 1H). Contractile responses to Ang II and to the adrenergic agonist phenylephrine were significantly increased in vessels from $s F l t 1$-treated pregnant mice compared with those with normal pregnancy $(P<0.05)$. These data confirm that exposure to sFLT1 is sufficient to induce the heightened vasoconstrictor and pressor response observed in preeclampsia. Removal of the vessel from contact with circulating sFLT1 during the ex vivo study suggests a persistent alteration in vascular function.

sFLT1 impairs vascular endothelial function independently of alterations in renin-angiotensin pathway. To evaluate mechanisms for Ang II sensitivity, we sought first to examine whether the reninangiotensin pathway was upregulated. Plasma renin and Ang II were assayed at gd17 in CMV-null pregnant mice compared with sFlt1-treated preeclamptic mice with higher blood pressure. sFlt1 mice had suppressed renin compared with CMV-null mice (Figure 2A; $P<0.05$ ), while no significant differences were found for plasma Ang II (Figure 2B). Ang II receptor (at1ra, at1tb, and at2r) mRNA expression was quantified in several candidate tissues over time in sFlt1-overexpressing compared with CMV-null mice. Ang II receptors were assessed in kidney (Figure 2C), mesenteric vessels (Figure 2D), uterine vessels (Supplemental Figure 2A), and uterus (Supplemental Figure 2B). We further assessed gene expression alterations of bradykinin receptor $\mathrm{B}_{2}(B d k r b 2)$ and endothelin-1 ( $\left.E d n 1\right)$ in both uterine and mesenteric vasculature (Supplemental Figure 2, C and D). Expression was dynamic over gestation; however, no differences were observed between CMV-null and sFlt1-overexpressing mice, indicating that alterations in Ang II receptors may not be responsible for the sensitivity noted in preeclampsia. Taken together with our murine studies (Figure $1 \mathrm{H}$ ) and prior human studies suggesting a general sensitivity to vasoconstrictors (4), these data do not support a direct role for alterations in circulating RAS to explain heightened sensitivity to Ang II in preeclampsia.

Impaired endothelial NO synthase and enhanced oxidative stress in preeclampsia-related Ang II sensitivity. Preeclampsia is characterized by decreased NO synthesis (21), and NO metabolites are inversely related to circulating sFLT1 concentrations (22). In the vasculature, endothelial NO synthase (eNOS) activity is the major regulator of NO production (23). VEGF and other growth factors are known to increase eNOS phosphorylation at serine 1177 (Ser1177), which is associated with increased enzyme activity (24-26). We therefore hypothesized that high sFLT1, an endogenous VEGF inhibitor, may block eNOS function and this could underlie Ang II sensitivity in preeclampsia. We examined protein expression of eNOS and its active form, phosphorylated eNOS (phospho-eNOS) (Ser1177), in cultured endothelial cells. As predicted, human endothelial cells exposed to VEGF demonstrated increased phosphorylation of Ser1177 residue on the eNOS protein in a dose-dependent manner that was inhibited in the presence of excess sFLT1 (Figure 3, A and B). We then examined both localization and expression of phospho-eNOS in mesenteric resistance vessels in pregnant sFlt1 mice or CMV-null mice. Positive phospho-eNOS signal was detected in arterial vascular endothelium. Robust signal intensity was observed in vessels from CMV-null mice, while phospho-eNOS expression was attenuated in sFlt1 mice (Figure 3C and Supplemental Figure 3A; $P<0.01$ ). Pregnant mice given L-NAME, a NOS inhibitor, developed Ang II sensitiv- 
A

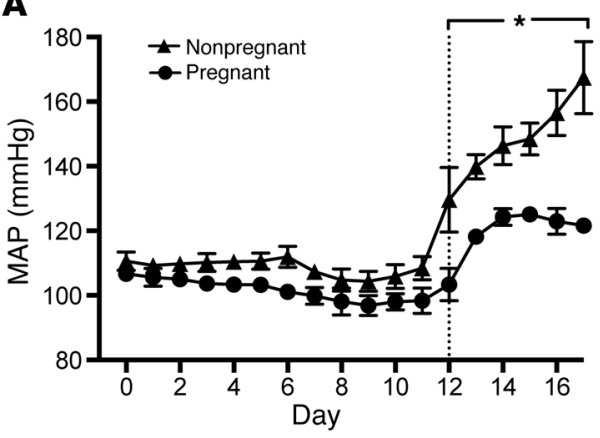

B

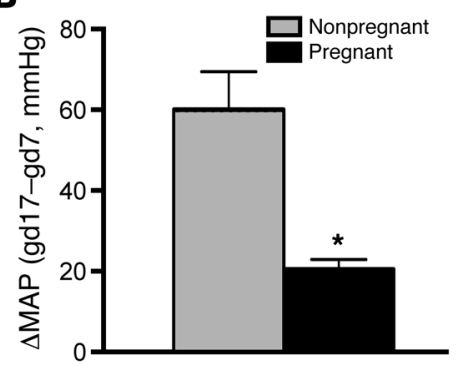

C

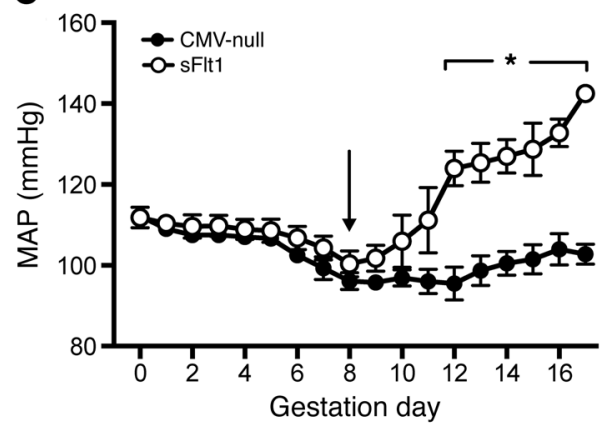

$\mathbf{F}$

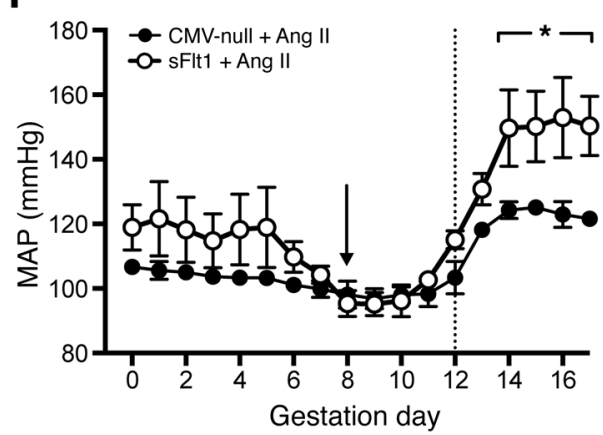

G
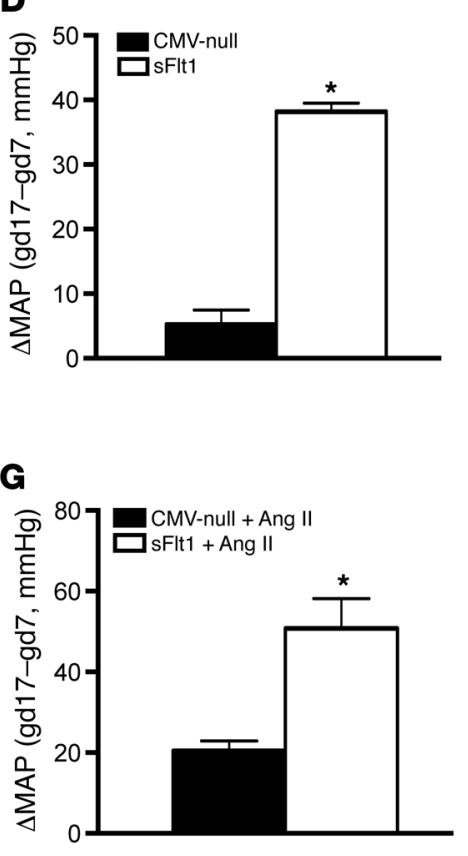

E

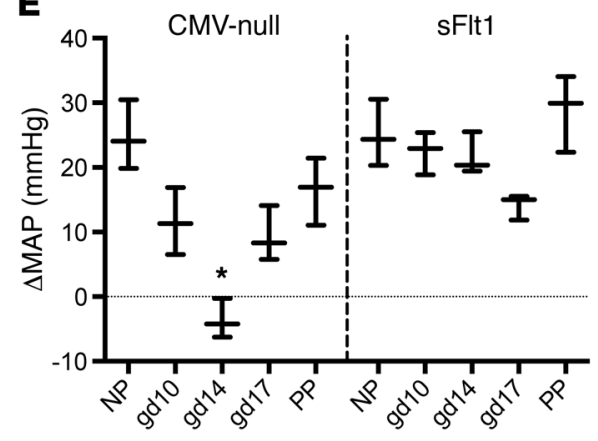

H

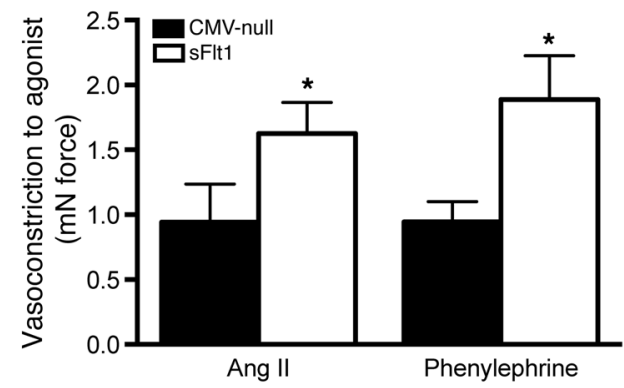

Figure 1. Ang II resistance characterizes normal pregnancy, while sFIt1 overexpression is sufficient to cause Ang II sensitivity. (A) Blood pressure measurements in nonpregnant $(n=5)$ and pregnant CD-1 mice $(n=3)$ with Ang II infusion via s.c. osmotic minipump beginning on day 12 . ${ }^{*} P<0.05$ for mean arterial pressure (MAP) in pregnant mice versus nonpregnant mice. (B) MAP comparison between nonpregnant $(n=5)$ and pregnant mice $(n=3)$ infused with Ang II. $\triangle$ MAP was obtained by subtraction of individual pressure at day 17 from that at day 7 . Day 7 was selected as the latest time point when all animals had no pressure differences (prior to intervention). ${ }^{*} P<0.001$ by unpaired 2-tailed $t$ test. (C) MAP of CMV-null $(n=5)$ and $s F I t 1$-injected $(n=3)$ mice over gestation. Mice were injected at gd8 (arrow). MAP in sFlt1 mice was significantly increased relative to CMV-null and within-group baseline beginning at gd12; ${ }^{*} P<0.001$. (D) Comparison of MAP between CMV-null mice $(n=5)$ and sFlt1 mice $(n=3)$. $\triangle$ MAP was obtained by subtraction of individual pressure at gd17 from that at gd7. ${ }^{*} P<0.01$ by 1-way ANOVA and Bonferroni's post-hoc test. (E) $\triangle$ MAP following acute i.v. Ang II bolus ( $30 \mathrm{ng}$ in $30 \mu \mathrm{l}$ saline plotted as medians (minimum and maximum), $n=3-5$. $\triangle$ MAP was calculated by subtraction of individual data points from preinjection data $(t=0)$ for each animal every 5 minutes for 15 minutes following injection. Data were recorded as nonpregnant (NP), at increasing gestational days, and postpartum $(P P)$. ${ }^{*} P=0.01$ compared with NP and sFlt1 at gd14. (F) MAP of CMV-null $(n=3)$ and sFlt1-injected $(n=4)$ mice given chronic Ang II over gestation. Mice were injected with adenovirus at gd8 (arrow) and implanted with s.c. osmotic minipumps containing Ang II at gd12 (dashed line). ${ }^{*} P<0.01$ for MAP in sFlt1 mice versus CMVnull mice. Data for CMV-null mice in this figure were obtained from data shown as the pregnant group in $\mathbf{A}$. (C) Comparison of MAP between CMV-null ( $n=3$ ) and sFlt1 mice $(n=4)$ given chronic Ang II. $\triangle$ MAP was obtained by subtraction of individual pressure at gd17 from that at gd7. ${ }^{*} P<0.05$ by 1 -way ANOVA with Bonferroni's post-hoc test. Data for the CMV-null mice in this figure were obtained from data shown as the pregnant group in $\mathbf{B}$. (H) Contraction of ex vivo mesenteric resistance vessels in gd17 CMV-null or sFlt1 mice in response to Ang II or phenylephrine ( $n=4$ per group). ${ }^{*} P<0.05$ by unpaired 2 -tailed $t$ test. Analyses used 2-way ANOVA with repeated measures, with the exception of $\mathbf{B}, \mathbf{D}, \mathbf{C}$, and $\mathbf{H}$, as indicated. All data represent the mean \pm SEM, except in $\mathbf{E}$.

ity similar to that observed in sFlt1-treated mice (Figure 3, D and E). Administration of L-NAME (with or without Ang II) did not alter plasma sFLT1 levels, which remained within normal pregnant range (Figure 3E; right $y$ axis). However, L-NAME-treated pregnant mice given vehicle did not develop hypertension, which is consistent with prior data in pregnant eNOS-deficient mice (Figure 3D) (27). These findings suggest that genetic and hor- monal factors, as well as Ang II, work in concert with diminished NO to induce hypertension during pregnancy. We next examined indicators of vascular damage in aortic sections from pregnant CMV-null mice, pregnant sFlt1-expressing mice, or L-NAMEtreated pregnant mice (Figure 3F). sFlt1- or L-NAME-treated mice demonstrated increased vascular oxidative stress as measured by positivity for MitoSOX Red and nitrotyrosine immunoreactivity in 

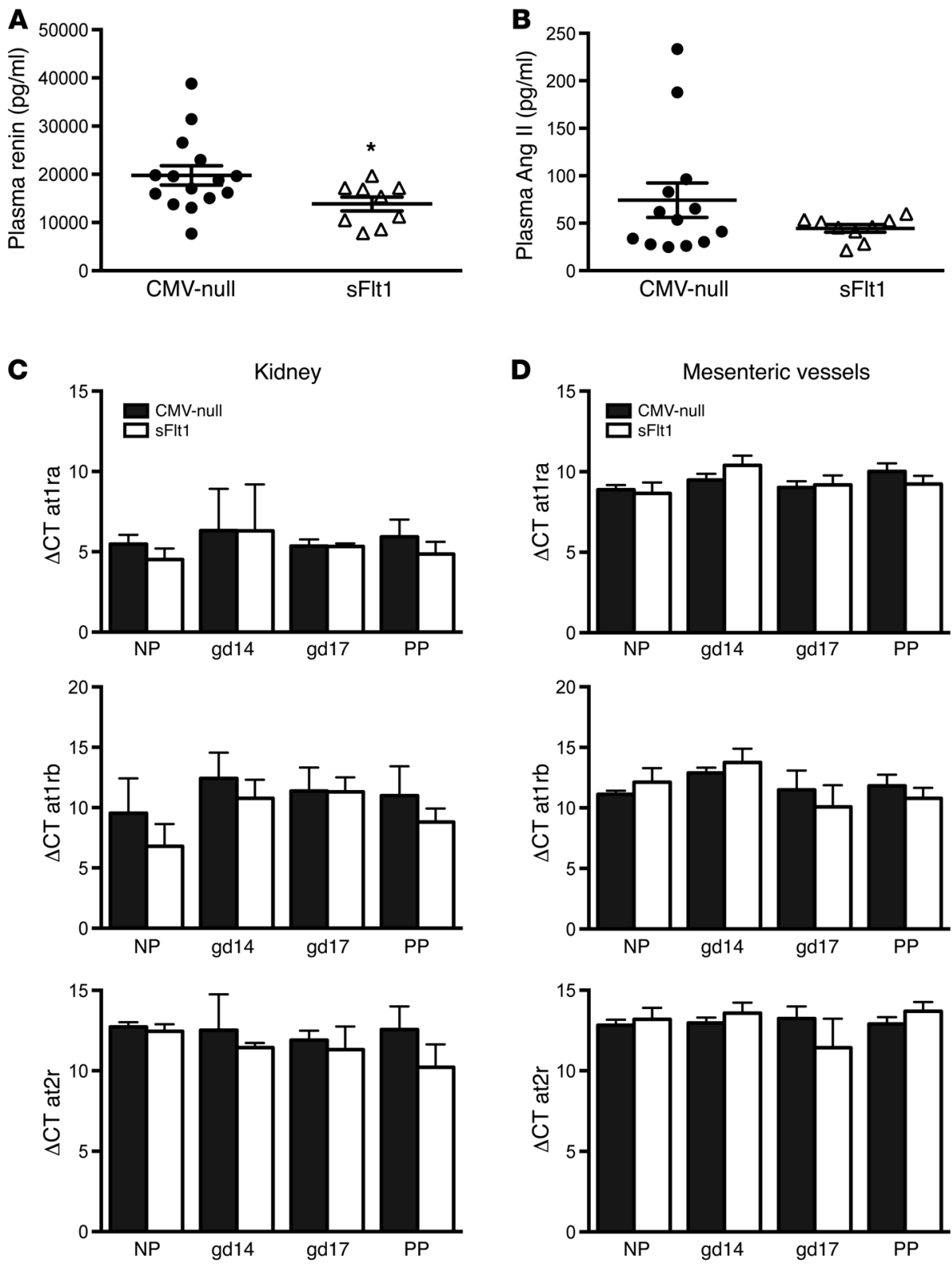
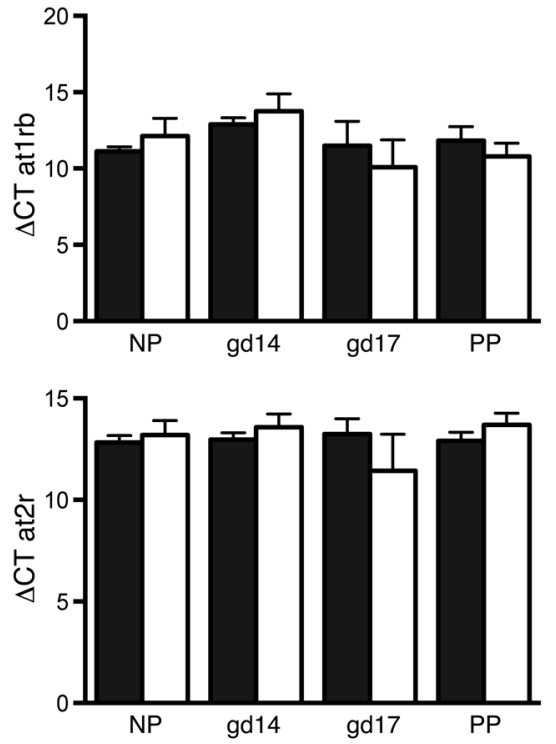

Figure 2. Assessment of the renin-angiotensin system in mice overexpressing sFlt1. (A and B) ELISA of plasma renin (A) and Ang II (B) from gd17 CMV-null $(n=14)$ and $\operatorname{sFlt1}(n=9)$ pregnant mice. Unpaired 2-tailed $t$ test; ${ }^{*} P<0.05$. (C and $\left.\mathbf{D}\right)$ Gene expression analysis of renal tissue (C) and mesenteric vessels (D) obtained from CMVnull and sFlt1 mice as nonpregnant (NP), gd14, gd17, and postpartum (PP). mRNA analysis (quantitative RT-PCR) was completed for the Ang II receptors (at1ra, at1rb, and $a t 2 r$ ). All data were normalized to $\beta$-actin ( $\Delta \mathrm{Ct})$. Comparisons between groups of NP, gd14, gd17, and postpartum (PP) mice revealed no differences. Nonpregnant mice were injected with adenovirus at least 7 days before collection. $n=3-8$ mice per time point were assayed per tissue. All data represent the mean \pm SEM. the intima and media (Figure 3F and Supplemental Figure 3, B and C). These data are indicative of greater vascular ROS production or reduced capacity for oxidant scavenging that is consistent with prior data in animal and cell culture studies demonstrating a key role for NO as a scavenger of oxidants $(28,29)$. These findings suggest that exposure to sFLT1 during pregnancy results in impaired eNOS function and increased oxidative stress within the smooth muscle media of the vasculature. Furthermore, L-NAME administration during pregnancy also induces Ang II sensitivity and oxidative stress, suggesting that sFLT1-induced eNOS dysfunction may be a primary mechanism of the increased Ang II sensitivity and oxidative stress observed in preeclampsia.

Inhibition of phosphodiesterase type 5 rescues sFLT1-induced hypertension and Ang II hypersensitivity. NO promotes vasodilation by activating smooth muscle guanylate cyclase to produce cGMP, which is then degraded by phosphodiesterases (PDEs). To determine whether sFlt1 overexpression directly impairs endothelial function via NO depletion, we explored whether enhancing cGMP with the PDE5 inhibitor sildenafil citrate (FDA-approved for treatment of pulmonary hypertension and erectile dysfunction) would prevent the adverse vascular effects of sFLT1 in pregnant mice. Preeclampsia was induced in pregnant mice by sFlt1 adenovirus (as above), and mice were randomized to sildenafil in drinking water or control beginning on gd8. Dams treated with sFlt1 and sildenafil remained normotensive throughout gestation and postpartum (Figure 4, A and B), relative to sFlt1-only dams. Plasma sFLT1 was not different between groups at gd17 (Figure 4B), indicating that sildenafil does not alter SFLT1 production. Despite similar sFLT1 levels, treatment with sildenafil significantly reduced contractile responsiveness of ex vivo mesenteric vessels from gd17 pregnant mice to Ang II (Figure 4C; $P<0.05$ ). Sildenafil also decreased aortic superoxide and nitrotyrosine immunoreactivity in mice exposed to sFLT1 during pregnancy (Figure 4, D and E). Urinary cGMP was elevated in animals receiving sildenafil (Figure 
A

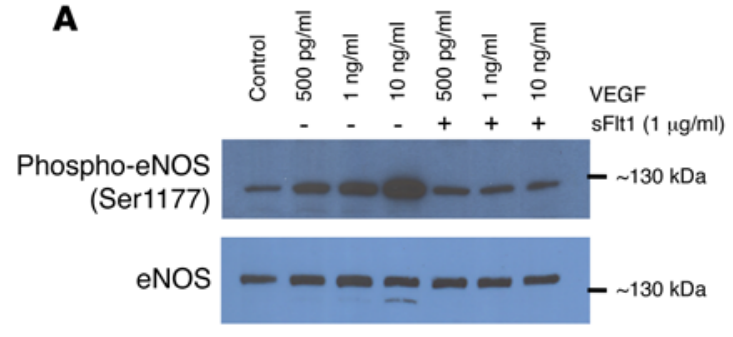

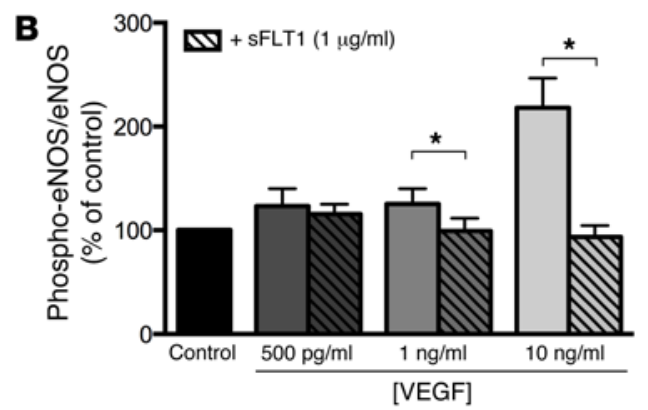
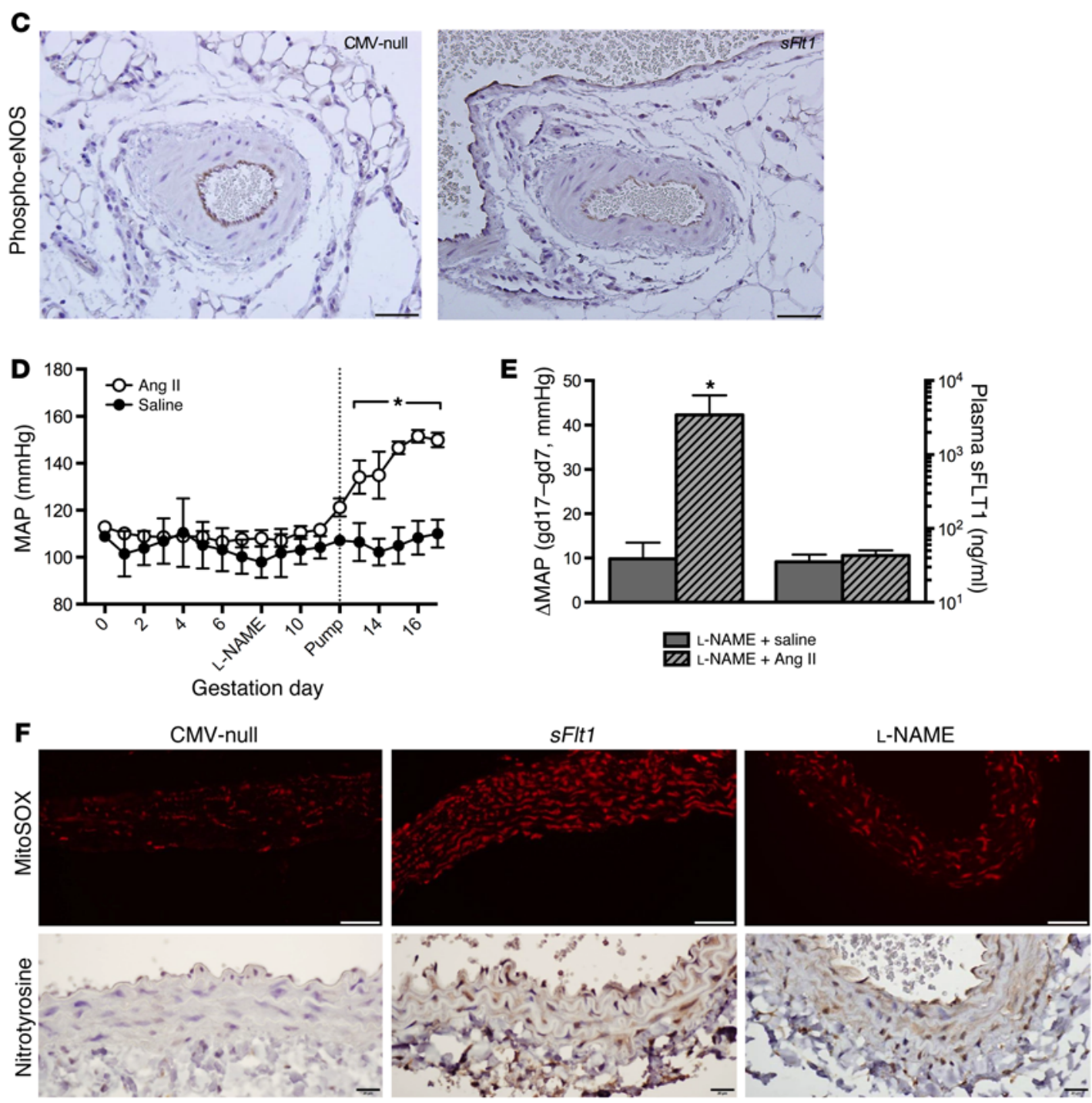

Figure 3. sFLT1 alters the vascular endothelium by decreasing eNOS function. (A) VEGF induces dose-dependent activation of eNOS in HUVECs, as measured by eNOS phosphorylation at Ser1177 (phospho-eNOS) relative to total eNOS by Western blotting. This is inhibited by excess sFLT1 (1 $\mu \mathrm{g} / \mathrm{ml}$ ). Representative of 4 individual experiments. (B) Densitometry of phospho-eNOS/total eNOS bands plotted as a percentage of control. VEGF-induced eNOS phosphorylation in HUVECs was approximately 2.5 -fold above control at $10 \mathrm{ng} / \mathrm{ml}$ of VEGF $(P<0.001 \mathrm{vs}$. all other groups). The increase in VEGF-induced phosphorylation was blocked when cells were preincubated with excess of $\mathrm{SFLT1}$; ${ }^{*} P<0.001$. Analysis by 2 -way repeated-measures ANOVA, $n=4$ individual experiments. (C) IHC for phospho-eNOS expression in mesenteric resistance vessels from gd17 pregnant mice given CMV-null (left) or sFlt1 (right). Images are representative of 4-5 mice per group. Scale bar: $100 \mu \mathrm{m}$. (D) Administration of L-NAME in drinking water during pregnancy phenocopies sFlt1-induced Ang II hypersensitivity. Pregnant mice were given $1 \mathrm{~g} / \mathrm{l}$ L-NAME in drinking water at gd8 and then implanted with s.c. osmotic minipumps infusing Ang II or saline control at gd12. Blood pressure became significantly elevated only in mice given L-NAME and Ang II ( $n=4$ per group; daily mean \pm SEM). Two-way repeated-measures ANOVA, ${ }^{*} P<0.01$. (E) Comparison of MAP and plasma sFLT1 between L-NAME + saline and L-NAME + Ang $I I$ pregnant mice $(n=4$ per group). $\triangle M A P$ was obtained by subtraction of individual pressure at gd17 from that at gd7. Gd7 was selected as the latest time point when all animals had no pressure differences (prior to intervention, permitting comparison). ${ }^{*} P<0.01$. Analysis by 1-way ANOVA with Bonferroni's post-hoc test. (F) Representative images of aorta isolated from CMV-null (left), sFlt1 (middle), and L-NAME (right) mice at gd17 staining for MitoSOX Red (top; scale bar: $50 \mu \mathrm{m}$ ) and nitrotyrosine (bottom; scale bar: $20 \mu \mathrm{m}$ ). All data represent the mean \pm SEM. 
A

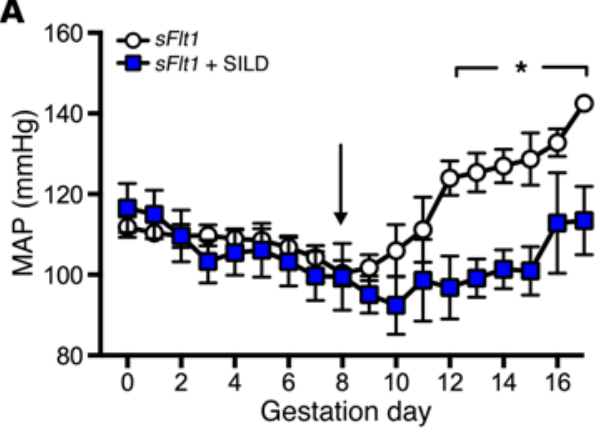

B

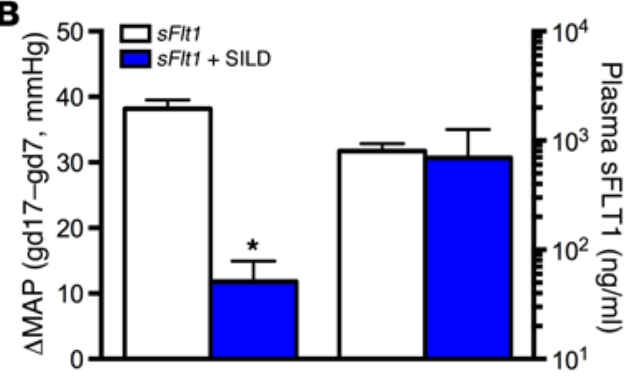

C

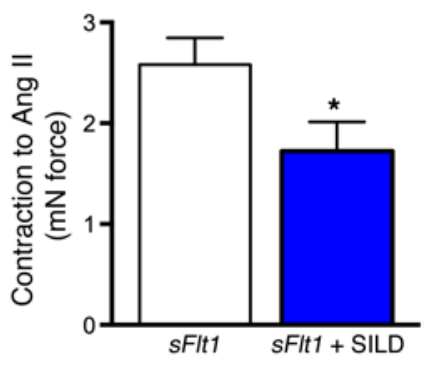

D

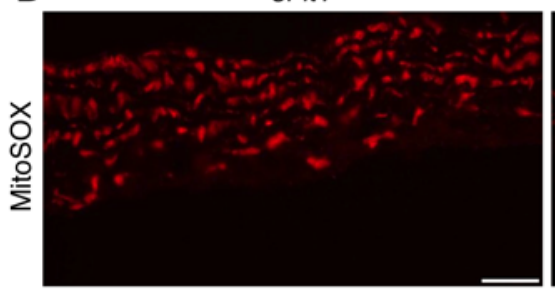

sFlt1

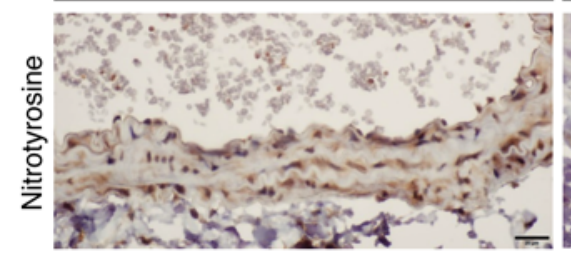

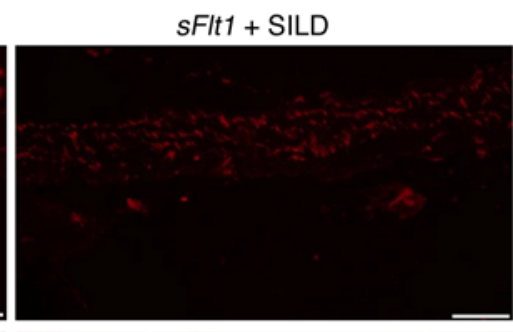

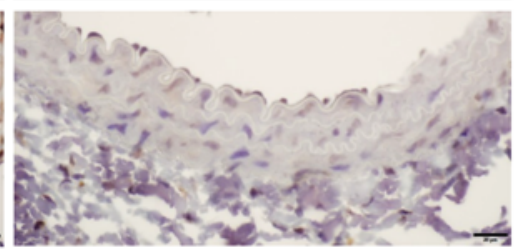

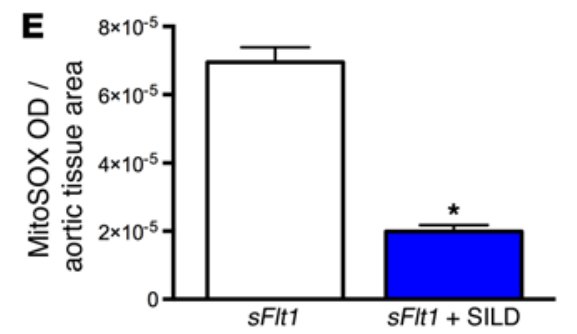

$\mathbf{F}$

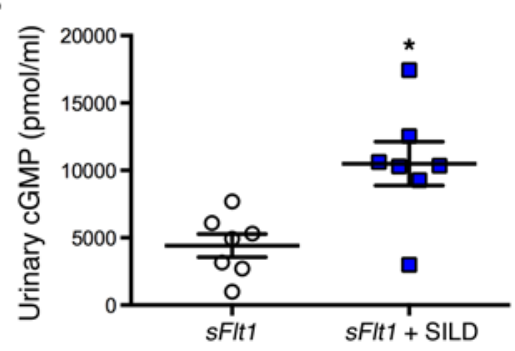

Figure 4. Augmentation of the cGMP pathway improves vascular outcomes in the sFIt1 overexpression model. (A) Pregnant mice given sFlt1 adenovirus at gd8 (arrow) with sildenafil citrate (sFlt1 + SILD, $n=5$ ) maintained normal blood pressure, whereas untreated mice developed hypertension $(n=3)$. ${ }^{*} P<0.001$, sFlt1 mice compared with earlier gestation days. Two-way repeated-measures ANOVA. Data for sFlt1 mice in this figure were obtained from data presented as sFlt1 group in Figure 1C. (B) Comparison of MAP and plasma sFLT1 levels between sFIt1 $(n=3)$ and sFIt1 + SILD mice $(n=5)$. $\Delta$ MAP was obtained by subtraction of individual pressure at gd17 from that at gd7. Gd7 was selected as the latest time point when all animals had no pressure differences (prior to intervention, permitting comparison). $\triangle M A P$ was significantly lower in sFIt1 + SILD-treated mice $\left({ }^{*} P<0.01\right)$, while plasma sFLT1 levels at gd17 were similar. Data for sFlt1 mice in this figure were obtained from data presented as sFlt1 group in Figure 1D. (C) Ex vivo contraction of mesenteric vessels in response to Ang II was restored by treatment with oral sildenafil in comparison with sFlt1 mice at gd17 ( $n=8$ per group). * $P<0.05$. (D) Representative images of aorta isolated from sFIt1 (left) and sFIt1 + SILD (right) mice at gd17 staining for MitoSOX Red (top; scale bar: $50 \mu \mathrm{m}$ ) and nitrotyrosine (bottom; scale bar: $20 \mu \mathrm{m}$ ). (E) Quantitation of MitoSOX Red immunofluorescence in aortic tissue from gd17 sFIt1- and sFIt1 + SILD-treated mice. OD per area (pixels ${ }^{2}$ ) of tissue was calculated in 5 high-power fields per sample ( $n=3$ aortas per group). ${ }^{*} P<0.001$ versus sFlt 1 by 2 -way ANOVA and Tukey's post hoc test. Data for sFIt1 mice in this figure were obtained from summary sFIt1 data presented in Supplemental Figure 3B. (F) Sildenafil treatment increased urinary cGMP compared with $s F I t 1$ alone. ( $n=7$ per group.) ${ }^{*} P<0.01$. Data represent the mean $\pm \mathrm{SEM}$, analyzed by unpaired 2 -tailed $t$ test with Welch's correction (except where noted).

$4 \mathrm{~F} ; P<0.01)$, suggesting that sildenafil was absorbed and had predictable pharmacodynamic effects. When normalized to urinary creatinine, the same trend is observed (data not shown).

Sildenafil improves fetal outcomes and improves placental perfusion. Due to enhanced vasoconstriction, preeclampsia is a condition of impaired placental blood flow that results in fetal growth restriction and risk of fetal loss (30). Thus, we next evaluated the safety and potential uteroplacental benefits of sildenafil in the $s F l t 1$ overexpression model. Pregnant mice were injected with $s$ Flt 1 at gd8, plasma sFLT1 was measured after 72 hours, and mice with similar $s F l t 1$ expression were paired and randomized to treatment (sildenafil) or control (water) at gd12. We first permitted the mice to deliver spontaneously to examine both premature delivery and live birth weight. Similarly to humans with preeclampsia, $s F l t 1$-overexpressing mice are prone to fetal losses or premature delivery (Supplemental Table 1). Live birth weights were measured the morning of delivery in mice treated with sFlt1 or $s F l t 1$ plus sildenafil ( $s F l t 1+$ SILD). Overall, birth weights were higher in $s$ Flt $1+$ SILD pups (Figure $5 \mathrm{~A}$ ). When separated into groups based on delivery date, sFlt1 + SILD pups were heavier, even if born prematurely (Figure 5B).

In order to examine placental weight and morphometry, a second study was conducted, and all dams were euthanized at gd17 (prior to spontaneous delivery). No significant changes were observed in fetal weights, litter size, or resorption rate (Supplemental Figure 4, A-E); however, placental weight increased in sFlt1 mice treated with sildenafil (Supplemental Figure 4B; $P<0.01$ ). As a proxy indicator of placental insufficiency, the ratio of the placental weight to the fetal weight was compared. This ratio was increased in $s F l t 1+$ SILD mice, implying that the heavier placenta 

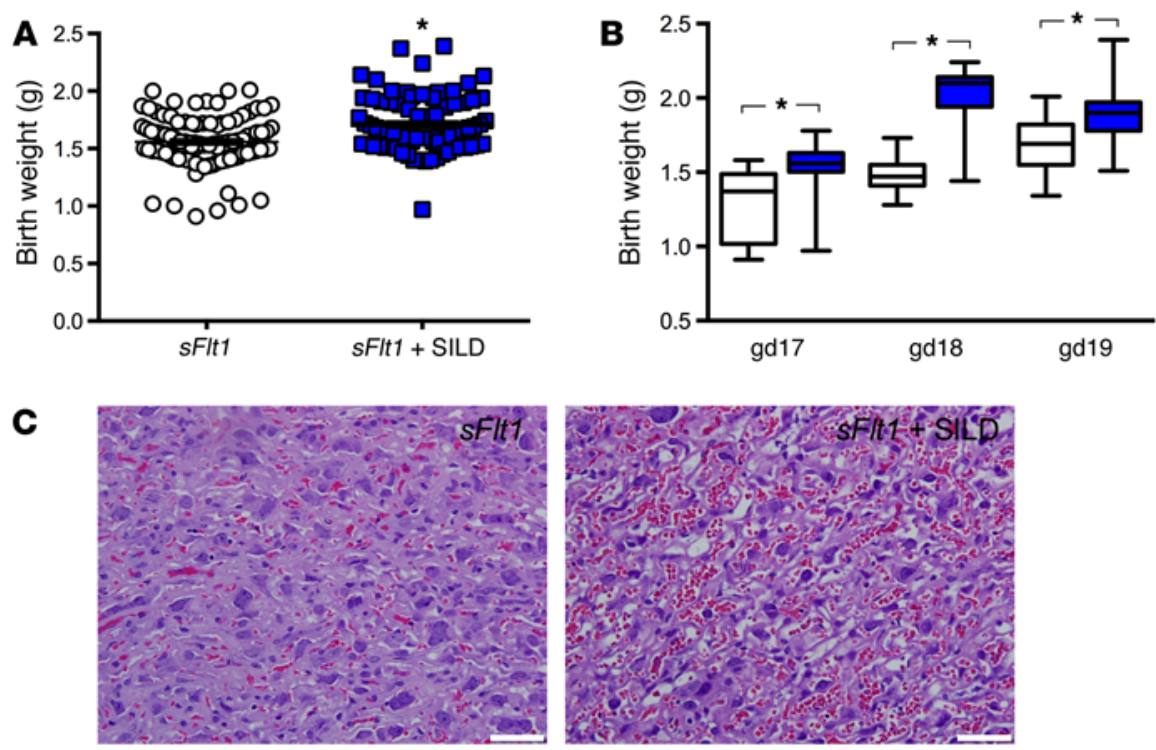

D

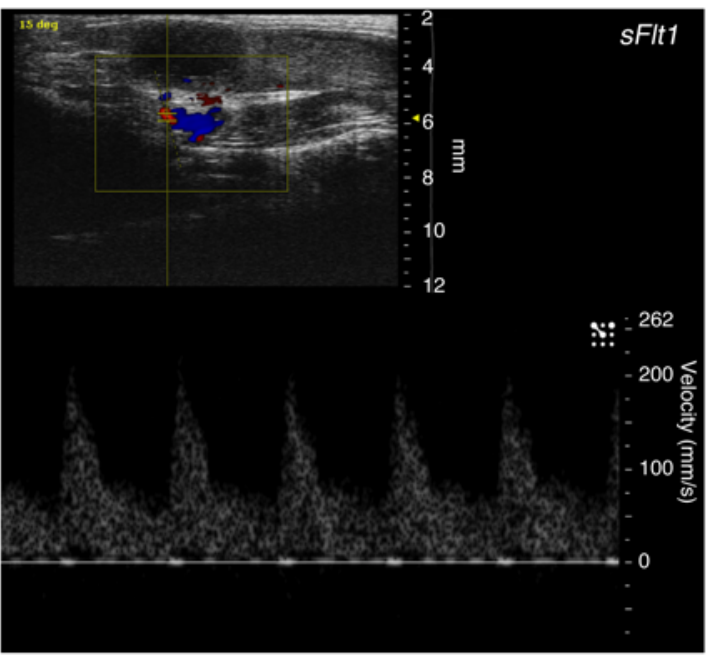

$\mathbf{E}$

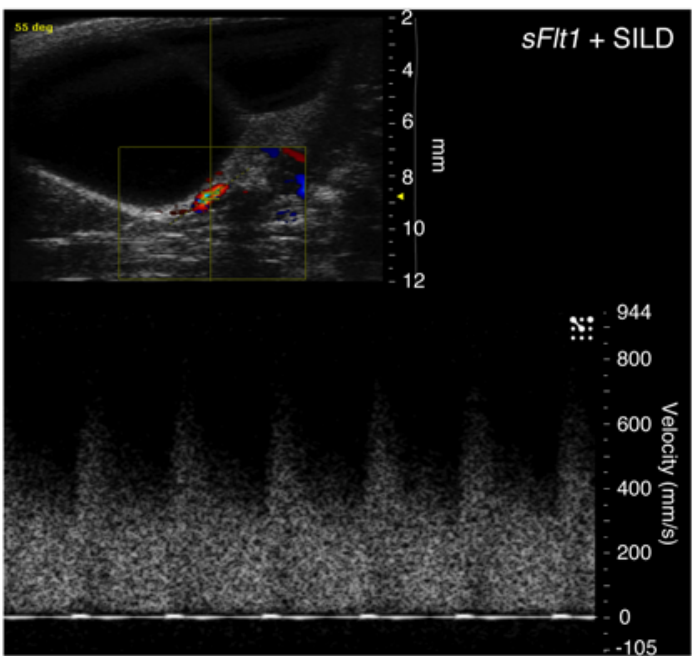

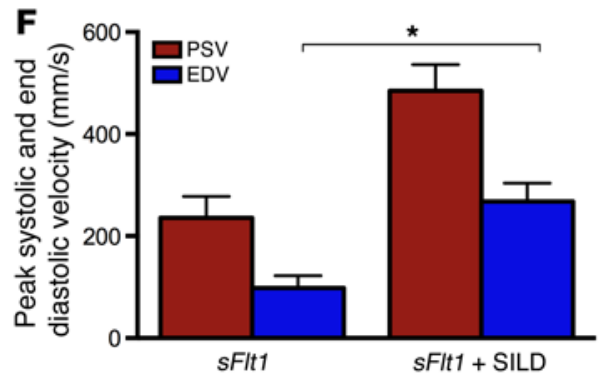

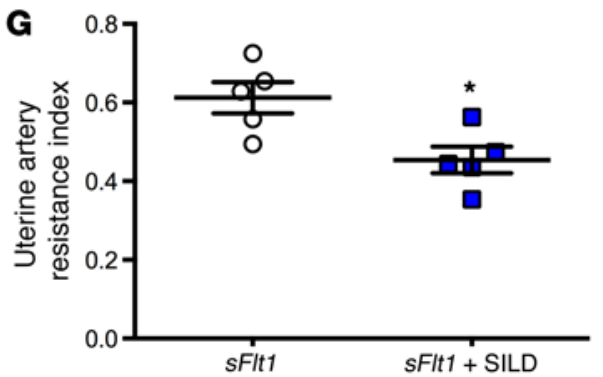

Figure 5. Sildenafil treatment in sFIt1-expressing dams improves fetal outcomes by enhancing uterine blood flow. (A) Live birth weights from mice injected at gd8 with sFlt1 adenovirus and randomized to sildenafil (sFIt1 + SILD, $n=7$ dams) or water ( $s F I t 1, n=8$ dams) at gd12. Plasma sFLT1 was similar prior to randomization. ${ }^{*} P<0.001$. (B) Live birth weights showing distribution by birth date (gd). Pups born to $s F l t 1+$ SILD dams ( $\left.n=7\right)$ had higher birth weights compared with those born to $s F I t 1$ dams $(n=8)$, independent of date of birth. ${ }^{*} P<0.001$. Data presented as boxplot (median, interquartile ranges, minimum and maximum), analyzed by 1-way ANOVA with Tukey's post-hoc test. (C) Placental histology from gd17 mice given sFIt1 or sFIt1 + SILD. Scale bar: $50 \mu \mathrm{m}$. (D and E) Representative Doppler waveform of the uterine artery at gd16 in sFlt1-treated (D) and sFlt1 + sildenafil-treated mice (sFIt1 + SILD) (E), highlighting differences in total flow (peak amplitude) and resistance (height of end diastolic velocity). (F) Uterine artery peak systolic velocity (PSV) and end diastolic velocity (EDV) obtained at gd16 via Doppler ultrasound ( $n=5$ per group). Sildenafil treatment increases EDV in comparison with $s F / t 1$-only mice. ${ }^{*} P<0.05$. (G) Uterine artery resistance index was reduced in the $s F I t 1+$ SILD group compared with $s$ FIt1 alone ( $n=5$ per group). Data are mean \pm SEM analyzed by unpaired 2 -tailed $t$ test, except where noted. ${ }^{*} P<0.05$. 

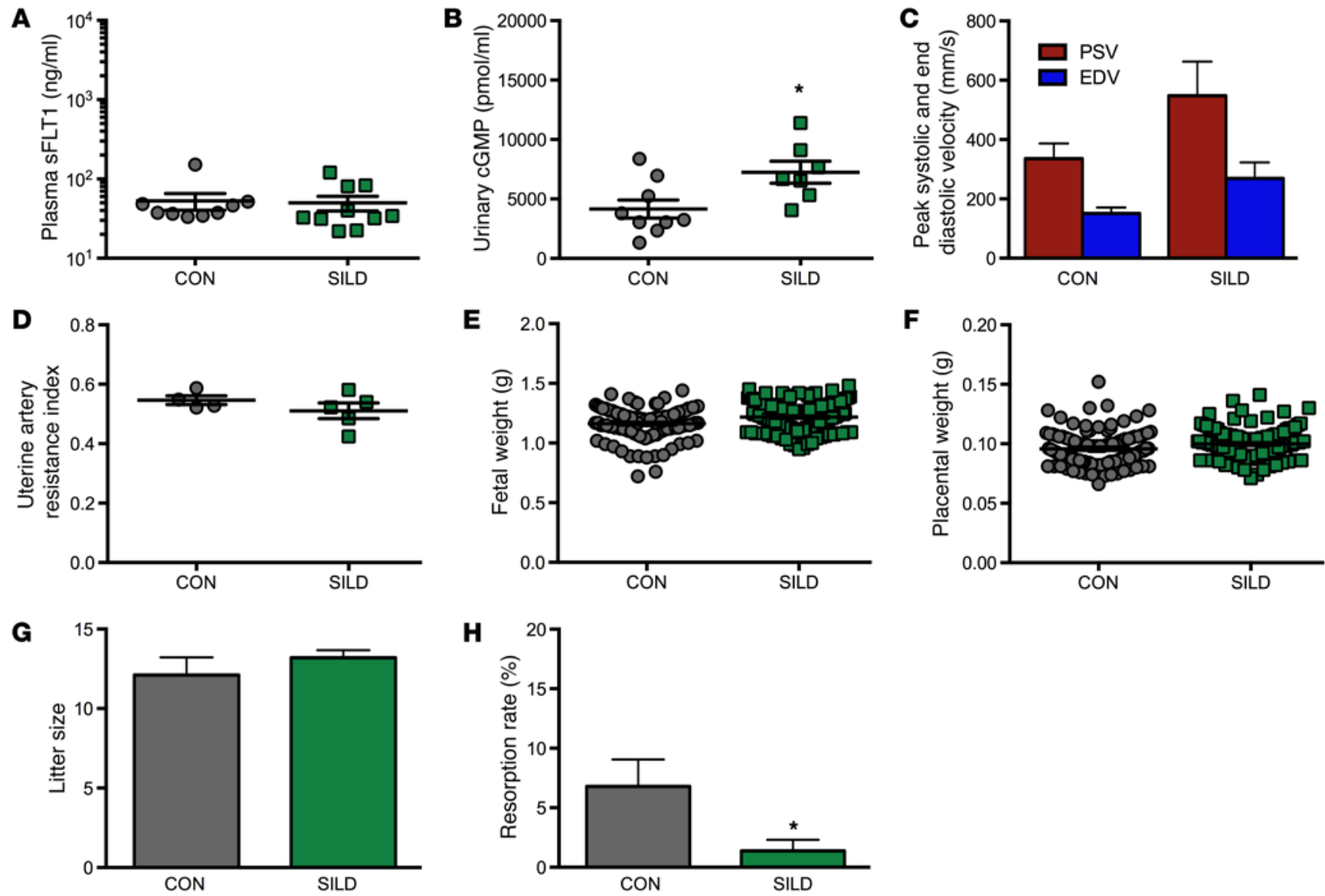

Figure 6. Sildenafil treatment in normal murine pregnancy. (A) Plasma sFLT1 measured at gd17 is not different between control (CON) and sildenafiltreated (SILD) mice. (B) Sildenafil treatment $(n=7)$ increased urinary cGMP compared with CON $(n=9)$. ${ }^{*} P<0.05$. (C) Uterine artery peak systolic and end diastolic velocities obtained at gd16 via Doppler ultrasound ( $n=4$ CON and $n=5$ SILD). (D) Uterine artery resistance indexes were not different between groups ( $n=4$ CON and $n=5$ SILD). (E and F) Fetal (E) and placental weights (F) from control ( $n=9$ litters) and SILD ( $n=10$ litters) dams at gd17. Sildenafil treatment increased both fetal $(P<0.001)$ and placental weight $(P<0.05)$. (C) Litter sizes were similar between CON and SILD mice. (H) Resorption rate was decreased in mice receiving sildenafil. ${ }^{*} P<0.05$, Mann-Whitney test. All data are mean \pm SEM, analyzed by unpaired 2-tailed $t$ test with Welch's correction, except $\mathbf{H}$. Unless specified, $n=9 \mathrm{CON}$ and $n=10$ SILD mice.

in this treatment resulted in improved function (Supplemental Figure 4C). Plasma sFLT1 levels were similar between groups at gd11 and gd17, with expected elevation at the later time point (Supplemental Figure 4F; $P<0.01$ ). We then examined placental morphology to confirm the presence or absence of pathological features. $s F l t 1$ treatment in pregnant mice was associated with reduced dilation of vascular spaces in the placenta, which was ameliorated by sildenafil treatment (Figure 5C). As in women with preeclampsia, mice with $s F l t 1$ overexpression had impaired uterine blood flow, as assessed via Doppler ultrasound. Sildenafil-treated sFlt1 mice had increased uterine artery end diastolic velocity compared with sFlt1 mice (Figure 5, D-F; $P<0.05$ ). Furthermore, sildenafil treatment significantly decreased the uterine artery resistance index of $s F l t 1$ mice with normalization of blood flow to the gravid uterus (Figure 5G; $P<0.05)$. These data are consistent with recent publications suggesting the efficacy of sildenafil in other models of fetal growth restriction or preeclampsia (31-34), and support the potential to use sildenafil as a novel therapy to alleviate signs and symptoms of preeclampsia. However, the safety and potential benefit of this therapy to the fetus have not been explored.
To explore safety and fetal effects in normal pregnancy, CD-1 mice were randomized to sildenafil or control at gd12. Plasma sFLT1 was not different between untreated (CON) and sildenafil-treated dams (Figure 6A). As expected, sildenafil increases urinary excretion of cGMP (Figure 6B; $P<0.05$ ). Doppler ultrasound of the uterine arteries was assessed at gd 16 and found that sildenafil treatment in normal pregnant mice does not impair blood flow or resistance index (Figure 6, C and D). At gd17, fetal (Figure 6E; $P<0.001$ ) and placental weights (Figure 6F; $P<0.05$ ) were increased with sildenafil treatment, while litter sizes were unaffected (Figure 6G). Interestingly, mothers treated with sildenafil had lower resorption rates than control mice (Figure 6H; $P<0.05$ ). Importantly, no maternal or fetal morbidities or mortalities were observed in the sildenafiltreated group. Together, these studies provide preclinical data supporting that PDE5 inhibition may be an effective strategy to circumvent sFLT1-induced endothelial dysfunction in preeclampsia, and that this therapy is safe in pregnancy and may improve both maternal and fetal outcomes in preeclampsia.

Impaired eNOS phosphorylation in placentas from preeclamptic women. We next examined the link between eNOS phosphorylation 
A

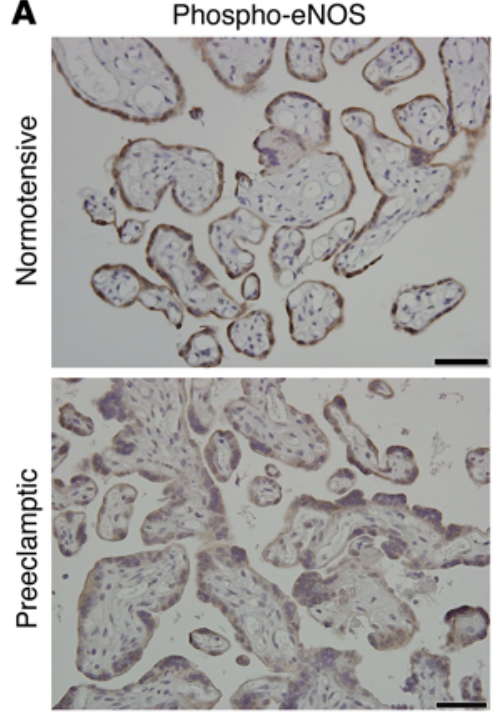

B

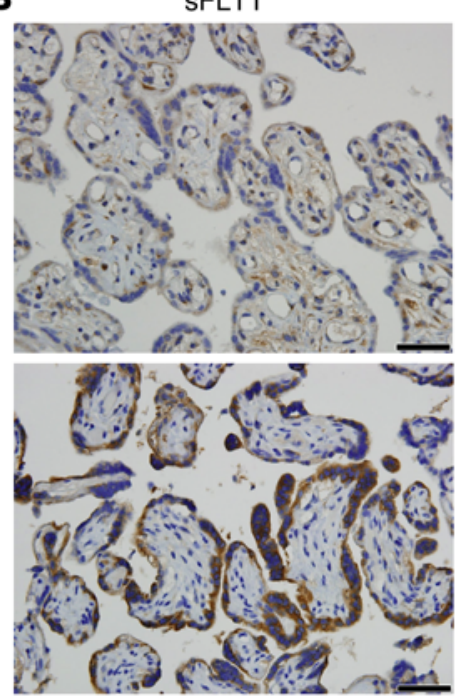

C

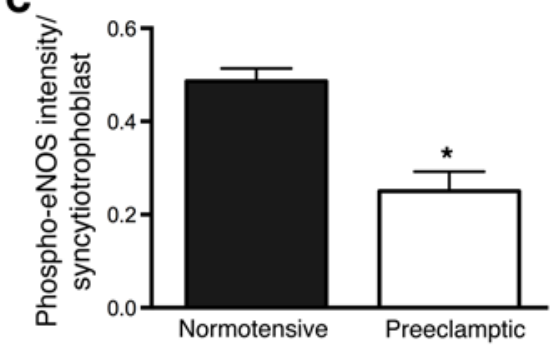

D

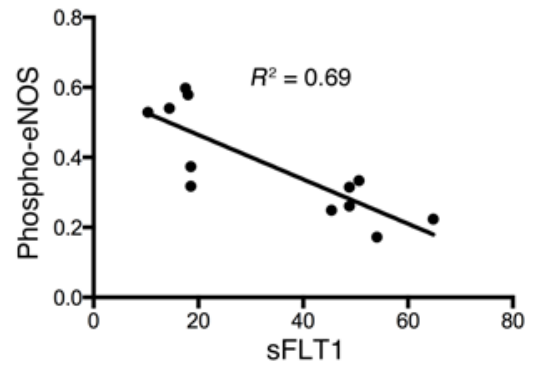

Figure 7. Phospho-eNOS and sFLT1 expression in human placentas from normotensive and preeclamptic women. (A and B) IHC of placentas obtained from preeclamptic women show decreased phospho-eNOS expression (A) and increased sFLT1 expression (B) in the syncytiotrophoblast layer compared with placentas from normotensive pregnancies. Bright-field microscopy images, representative of $n=6$ per group. Scale bar: $50 \mu m$. (C) Quantitation of phospho-eNOS immunoreactivity in placentas from uncomplicated and preeclamptic placentas. OD per area (pixels²) of tissue was calculated in 4 highpower fields per sample ( $n=6$ per group). ${ }^{*} P<0.001$, presented as the mean \pm SEM. Analyzed by 2 -way ANOVA. (D) Phospho-eNOS immunoreactivity correlates negatively with sFLT1 immunoreactivity in the placenta. Analyzed by Pearson's correlation, $P<0.001$.

and SFLT1 secretion in humans to determine the relevance of the mechanism identified in mice to the human condition. We examined placental histology from a cohort of preeclamptic subjects and uncomplicated gestations. IHC staining of phospho-eNOS was localized to the syncytiotrophoblast in placental samples from uncomplicated and preeclamptic subjects (Figure 7A). Adjacent sections were stained for sFLT1 expression (Figure 7B). Mean phospho-eNOS staining intensity was robust in normal pregnant samples and attenuated in preeclamptic samples (Figure 7C; $P<0.001$ ). As previously reported, sFLT1 staining was weak and sparse in normal placenta with over 3-fold upregulation in the syncytiotrophoblast layer of the preeclamptic placenta $(P<0.001)$. Correlation of mean staining intensities revealed a negative relationship between phospho-eNOS and sFLT1 expression in the placenta (Figure 7D; $\left.R^{2}=0.7 ; P<0.001\right)$. These data in human placentas are consistent with the model determined from our in vitro and in vivo observations in which elevated SFLT1 inhibits phosphorylation of eNOS in women with preeclampsia.

\section{Discussion}

In this article, we explored the mechanism and potential treatment of sensitivity to vasopressors in preeclampsia and report the following: First, overexpression of sFLT1, a soluble protein that is elevated in preeclampsia, is sufficient to induce vascular sensitivity to vasopressors (including Ang II) in pregnant mice. Second, sFLT1 acts on the maternal endothelium to inhibit eNOS phosphorylation, and this is associated with increased blood pressure, vessel oxidative stress, and sensitivity to vasopressors. Third, sildenafil, a PDE5 inhibitor that raises cGMP levels, is sufficient to restore Ang II resistance during pregnancy and inhibit sFLT1-mediated hypertension. Sildenafil also increased blood flow to the fetal-placental unit and improved fetal outcomes. This finding is unique and contrary to the placental effects of current antihypertensive therapies used in pregnancy, which are often associated with decreased uterine blood flow to the fetus, leading to further risk of fetal growth restriction (30). Finally, sFLT1 expression correlates inversely with eNOS phosphorylation in human placental tissue. These data support a model in which endothelial damage due to high circulating sFLT1 leads to dysfunctional eNOS signaling, which, in turn, promotes vascular oxidative stress and enhances sensitivity to vasoconstrictors (Figure 8) that results in maternal hypertension and fetal growth restriction. Taken together with prior clinical data that sFLT1 alterations antedate hypertension of preeclampsia (35), we suggest that ameliorating the toxic effects of sFLT1 acting downstream of eNOS may alleviate preeclamptic morbidity in the mother without adverse consequences to the fetus.

The modified vascular responses that occur during normal pregnancy that are prevented during hypertensive pregnancy complications have intrigued clinicians and researchers for decades. It was postulated that discerning the physiology observed in normal pregnancy could provide insight for improved treatment of many hypertensive disorders. While resistance to Ang II during normal pregnancy has been most studied, literature supports generalized insensitivity to vasoactive substances in normal pregnancy (5). Conversely, Ang II sensitivity during preeclampsia has become so carefully documented that it is a diagnostic method to predict future development of hypertension during pregnancy $(3,6)$. Studies in pregnant rodents have been instrumental in determining contributions to hemodynamic and vascular alterations (36, 37). Importantly, Ang II resistance observed during pregnancy has been found to be specific to vascular smooth muscle cells, and not to actions on the renal tubules (38). Together with data show- 


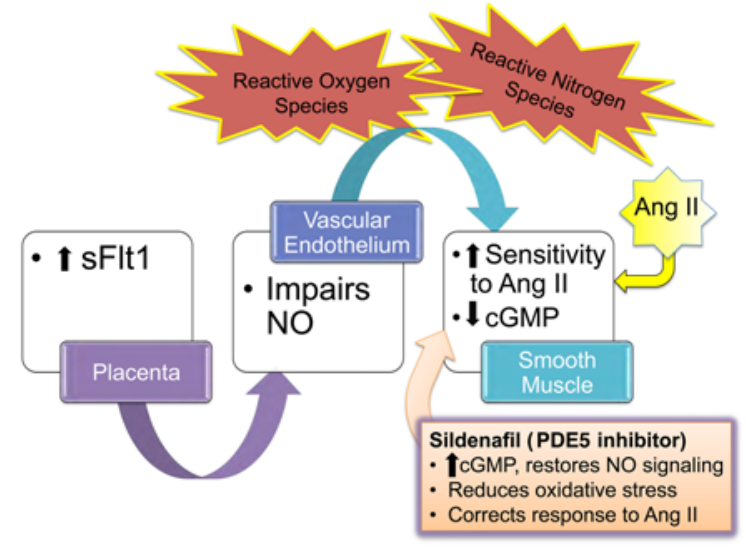

Figure 8. Summary model of findings. Our data are consistent with a model in which the preeclamptic placenta produces excess sFLT1, which acts on endothelial cells to impair NO production. This results in enhanced vascular oxidative stress and decreased smooth muscle cell cGMP, which contribute to enhanced vascular sensitivity to vasoconstrictors. In the maternal resistance vasculature this contributes to hypertension, and in the placental vasculature this decreases placental perfusion and contributes to fetal growth restriction or demise. Treatment with sildenafil, which increases smooth muscle cell cGMP, restores NO signaling, reduces oxidative stress, attenuates vasoconstriction, and improves maternal and fetal outcomes.

ing that in preeclampsia, there is increased vascular sensitivity to other vasoconstrictors, this suggests it is unlikely that alterations in circulating renin or Ang II underlie the pathogenesis of hypertension in preeclampsia. Our data indicate that heightened contractile responsiveness to pressors observed in preeclampsia is a secondary phenomenon that is induced by increased circulating sFLT1, which acts by inhibiting eNOS function. These findings are also consistent with impairment in NO production noted in VEGF pathway inhibitor-related hypertension in cancer subjects (39).

Several groups have also reported that sildenafil may be beneficial in other animal models of pregnancy complications, such as the rat model of placental ischemia, the Dahl salt-sensitive hypertensive rat, and the catechol- $O$-methyltransferase-deficient model of growth restriction $(31,33,40)$. Bolnick et al. have also reported that trophoblast invasion, a process that is defective in preeclampsia, may be promoted by sildenafil in culture studies (41). Our findings confirm the beneficial effects of sildenafil in an independent mouse model of preeclampsia and provide evidence that improvement in maternal hypertension by sildenafil is associated with better placental perfusion and improved fetal weights. We also suggest that sildenafil may be acting by reversing the Ang II sensitivity and ameliorating vascular oxidative stress. Preeclampsia develops in stages over time with vascular responsiveness to Ang II antedating clinical signs of preeclampsia. The time course of Ang II hypersensitivity closely resembles the increasing pattern of circulating sFLT1 in preeclampsia $(3,17)$. On the basis of our data demonstrating that sildenafil prevents the development of vascular oxidative stress and enhanced vasoconstriction in mice exposed to sFLT1, it might be expected that initiation of sildenafil during subclinical preeclampsia (using enhanced pressor response and elevated plasma sFLT1 as biomarkers) would be far more beneficial to the mother and the fetus than during overt maternal hypertension. It is therefore not surprising that in a small clinical trial, sildenafil given to women with established preeclampsia was not associated with improvement in pregnancy outcomes (42). Furthermore, since preeclampsia is a very heterogeneous disease, it may be critical to focus therapeutic studies with sildenafil specifically in subgroups of patients who have augmented circulating sFLT1 and increased pressor response to Ang II.

In normal pregnancy, despite enhanced Ang II and volume expansion, NO production is amplified (21, 43-47), likely by growth factors such as VEGF and placental growth factor that are required for normal placental angiogenesis $(24,25,48)$. The actions of NO to reduce blood pressure during pregnancy are pleiotropic, primarily through endothelium-dependent dilation and resulting smooth muscle relaxation, which decreases vascular resistance. NO also negatively regulates the activity of many vasopressors, including endothelin-1 and Ang II (49-51), and is antithrombotic (52). Our study and others, including in vivo studies of pregnant eNOS mutant mice and rodents given L-NAME during pregnancy, provide valuable experimental evidence that inhibition of NO during pregnancy induces hypertension, similar to preeclampsia $(34,36,50,53,54)$. Decreased NO bioavailability can also lead to hypertension by enhancing vascular oxidative stress, as NO is the principal scavenger of oxidants in the vasculature (55). Vascular oxidative stress exacerbates vasoconstriction, and there is ample evidence that angiotensin-induced hypertension requires oxidants produced in the vasculature (56). Our finding that interference with NO signaling by sFLT1 leads to enhanced vascular sensitivity to pressors that can be reversed by sildenafil also has relevance outside of pregnancy. Vascular endothelial dysfunction is characterized by impaired NO production and is an early manifestation of numerous systemic pathologies, including diabetes and atherosclerosis, and is a major contributor to cardiovascular and cerebrovascular disease (57). Genetic polymorphisms in VEGF and its receptors have been associated with susceptibility to coronary heart disease (58). Also, cancer patients treated with novel anti-VEGF pathway inhibitors almost universally develop hypertension and a preeclampsia-like syndrome $(59,60)$. It will be important to determine whether similar mechanisms of enhanced vasoconstriction and Ang II sensitivity are involved and whether sildenafil or other drugs downstream of NO may be novel therapeutic strategies for endothelial dysfunction and hypertension in these other clinical scenarios.

Our article leaves many unanswered questions and paths for future work. We do not know whether other pathways that are downstream of VEGF signaling such as endothelial prostacyclins and hydrogen sulfide may also contribute to the sFLT1-mediated Ang II sensitivity and hypertension (61). We also do not know whether other molecules known to be upregulated in human preeclampsia enhance the effects of sFLT1 on vascular function. For example, soluble endoglin, which is also elevated in preeclampsia, may contribute to vascular sensitivity to vasopressors by affecting eNOS enzyme at other activation sites (62) or by other mechanisms. It would also be important to evaluate the effects of angiotensin receptor autoantibody (AT1-AA) in the context of high sFLT1. While suppression of circulating RAS is typical (63), there is ample evidence that AT1-AA is present in patients with preeclampsia $(7,64-66)$. We also do not know the mechanisms of 
Ang II resistance during normal pregnancy that is maximal during mid-gestation, but we speculate that vasodilatory factors such as relaxin and other growth factors that induce NO may be responsible. Finally, we do not know whether high circulating sFLT1 and the associated impairment of eNOS function and increased oxidative stress during pregnancy contribute to vascular changes that persist into the postpartum period, including epigenetic changes and persistent Ang II sensitivity that may predispose to long-term cardiovascular risk in women exposed to preeclampsia $(67,68)$.

In summary, our data demonstrate that pharmacological inhibition of PDE5 with sildenafil in the setting of sFLT1-induced hypertension and vascular dysfunction in pregnancy restores the actions of endothelial NO: this appears to be a final common pathway in the pathogenic mechanism of preeclampsia and associated Ang II sensitivity. With NO production restored, there is decreased vascular oxidative stress, improved vascular function, and normalization of blood pressure in the mother. This, in turn, increased uteroplacental blood flow, permitting optimal nutrient availability to the fetus (69) with improved fetal growth and survival. These findings may also have important implications in the pathogenesis of VEGF pathway inhibitor-induced hypertension in cancer patients and of Ang II sensitivity in nonpregnant states such as in women with a history of prior preeclampsia (70-72), major risk factors for cardiovascular disease. Prospective clinical trials are needed to evaluate the beneficial effects of sildenafil started in women with early biomarker evidence of preeclampsia in the prevention of preeclampsia-related morbidity.

\section{Methods}

Additional methods can be found in the Supplemental Methods online.

Mice. Adult male and female CD-1 [Crl:CD1(ICR)] mice were purchased from Charles River Laboratories. Animals were housed in a temperature- and humidity-regulated environment with a 12-hour light cycle. For all timed pregnancy experiments, virgin female mice (8 to 12 weeks old) were placed with a stud male overnight. Observation of a copulation plug the following morning was denoted gestational day 0 (gd0). Plugged females were weighed and removed from the stud cage. Pregnant females were injected on gd8 with either murine sFlt1 adenovirus or CMV-null adenovirus (control pregnant) diluted in sterile saline via tail vein under isoflurane anesthesia, as described previously (18). In CD-1 pregnant mice, live birth typically occurs at gd18-19, compared with gd19-20 for inbred strains (Supplemental Table 1). sFlt1 overexpression can result in embryo/fetal resorption, miscarriage, or premature delivery depending on the dose and duration of exposure of sFLT1 protein (19). sFlt1 adenovirus dose was titrated on the basis of batch efficacy to provide plasma sFLT1 values 100-750 ng/ml, which result in hypertension phenotype without significant fetal resorption (73).

Alternatively, pregnant mice were given $N_{\omega}$-nitro-L-arginine methyl ester hydrochloride (L-NAME, a NOS inhibitor; SigmaAldrich) in drinking water at gd8. Dose of L-NAME was calculated as $100 \mathrm{mg} / \mathrm{kg} / \mathrm{d}(1 \mathrm{~g} / \mathrm{l})$. Mice were sacrificed as nonpregnant or at gd14, gd17, or 72 hours postpartum via cardiac puncture under deep isoflurane anesthesia, and organs were collected for vascular reactivity studies, histology, protein, and RNA.

Blood pressure measurement. Direct blood pressure monitoring was done using radiotelemetry (Data Sciences International). Surgi- cal implantation of TA11PA-C10 transmitters was performed as previously described (74). Briefly, 10-week-old female CD-1 mice were anesthetized with inhaled isoflurane in $\mathrm{O}_{2}$, with meloxicam $(5 \mathrm{mg} / \mathrm{kg}$, s.c.) as analgesic. Mice were permitted 1 week to recover from surgical implantation, followed by data acquisition for $3-5$ days to assess data quality and establish normal blood pressure (nonpregnant) values. Mice were then mated (as above) and recorded throughout pregnancy (74). Blood pressure data were averaged daily per animal.

Acute or chronic Ang II infusion studies. In the acute studies, Ang II (30 ng in $30 \mu \mathrm{l}$; Sigma-Aldrich) was injected i.v. to awake, unrestrained, telemetry-implanted mice during recording. Bolus injections were administered up to 3 times to nonpregnant mice and once on gd10, gd14, and gd17, and 72 hours postpartum. Analysis of acute Ang II response used blood pressure/heart rate normalized before injection $(t=0)$ every 5 minutes for 20 minutes, presented as moving average of the change relative to $t=0$.

In chronic studies, telemetry mice that had received either $s F l t 1$ or null adenovirus at gd 8 were surgically implanted with s.c. osmotic minipumps at gd12 (model 1002; Alzet) containing Ang II (0.43 mg/ $\mathrm{kg} / \mathrm{d}$ ) diluted in sterile saline. Pumps were filled and implanted according to the manufacturer's directions. Chronic Ang II response was also assessed in nonpregnant mice to compare with normal pregnancy. Nonpregnant telemetry mice were recorded for minimally 14 days before implantation of s.c. minipumps (as above) filled with Ang II. During L-NAME experiments, telemetry mice were given L-NAME in drinking water at gd8 (in lieu of $s F l t 1$ adenovirus), and then implanted at gd12 with s.c. minipumps containing Ang II or sterile saline alone.

Sildenafil citrate studies. Experiments using sildenafil citrate (Cayman Chemical) for prevention of preeclamptic-like signs were conducted in telemetry-implanted mice, as above. In these animals, 100 $\mathrm{mg} / \mathrm{kg} / \mathrm{d}$ sildenafil was provided in the drinking water immediately following adenovirus injection (gd8), and animals were sacrificed at gd17 to evaluate preeclampsia phenotypes. In other studies, pregnant mice were injected with $s F l t 1$ adenovirus at gd8. Seventy-two hours later (gd11), plasma was obtained and assayed for circulating sFLT1 protein. Mice were paired and randomized to treatment (sFlt1 + SILD) or normal water (sFlt1) on gd12 based on similar elevations in circulating sFLT1 protein. At gd17, mice were euthanized for vascular reactivity studies or tissue collection. In a separate study, paired mice were permitted to deliver spontaneously to permit assessment of preterm delivery and live birth outcomes.

An additional set of experiments was conducted to more thoroughly examine the impact of sildenafil on maternal and fetal outcomes. Pregnant mice were assigned to no treatment (CON) or sildenafil treatment (SILD) from gd12 to determine the impact of treatment on normal pregnancy. All groups had Doppler ultrasound of the uterine arteries at gd16 and were euthanized at gd17 for measurement of fetal and placental weights.

Western blot analysis. Eighty to ninety percent confluent HUVECs (ATCC) were incubated in $0.25 \%$ serum/EGM-2 medium without additional growth factors overnight. Cells were stimulated with increasing doses of recombinant VEGF-165 (R\&D Systems) in the presence and absence of $1 \mu \mathrm{g} / \mathrm{ml}$ recombinant human sFLT1 (R\&D Systems) for 15 minutes in EGM-2 medium without serum. Cells were washed with cold $\mathrm{PBS}$ and lysed with $1 \times$ cold cell lysis buffer supplemented with $1 \mathrm{mM} \mathrm{Na}_{8} \mathrm{VO}_{4}, 10 \mathrm{mM} \mathrm{Na}_{4} \mathrm{P}_{2} \mathrm{O}_{7}, 25 \mathrm{mM} \mathrm{NaF}$, and protease inhibitors (Cell Signaling Technology). Cell lysates were quantified for 
protein concentrations, and $50 \mu \mathrm{g}$ protein was separated in $8 \%$ SDSPAGE and immunoblotted with antibodies against phospho-eNOS (Ser1177) (07-428; EMD Millipore) or antibody that recognizes total eNOS (07-520; EMD Millipore). Densitometry was performed using UN-SCAN-IT gel software (Silk Scientific).

Human subjects. Details regarding preeclampsia diagnosis and placental collection have been published (75). All subjects presented to Beth Israel Deaconess Medical Center for delivery. Placental biopsies were obtained within 30 minutes of delivery and placed in 10\% neutral buffered formalin for routine tissue processing.

Histology and IHC. Mouse tissues collected for histology and IHC were immediately placed in $10 \%$ neutral buffered formalin for routine processing and staining. Human placental tissue was obtained from uncomplicated or preeclamptic pregnancies for IHC of phospho-eNOS. Histopathology of mouse kidneys was conducted to assess "glomerular endotheliosis" as described previously (18). For nitrotyrosine IHC of the abdominal aorta, 5 - $\mu \mathrm{m}$ paraffin sections were cut and deparaffinized. Slides were rinsed in PBS followed by a 15 -minute quenching of endogenous peroxidase with $3 \%$ hydrogen peroxide in distilled water. To block nonspecific binding, slides were incubated at $37^{\circ} \mathrm{C}$ with $2.5 \%$ normal horse serum in PBS for 40 minutes. Rabbit polyclonal nitrotyrosine antibody $\left(\mathrm{A}-21285 \operatorname{IgG}_{2} \mathrm{~b}\right.$; Thermo Fisher) was used at 1:500 overnight at $4^{\circ} \mathrm{C}$. Slides were then rinsed in PBS and developed using ImmPRESS HRP Anti-Rabbit IgG (Peroxidase) Polymer Detection Kit, according to directions (Vector Laboratories). IHC staining for phospho-eNOS was completed on mesenteric resistance vessels from mouse and term human placenta. Slide preparation and blocking methods were similar to those for nitrotyrosine, with an antigen retrieval step in citrate buffer, according to the manufacturer's recommendations (Vector Laboratories). Rabbit polyclonal phospho-eNOS (Ser1177) from Cell Signaling Technology $(1: 100 ; 9570)$ was used for mouse samples and from Abcam (1:1,000; ab87750) for human samples. Development for visualization used VECTASTAIN Elite ABC Kit (Rabbit IgG; PK-6101; Vector Laboratories), according to the manufacturer's recommendations. Serial sections of human placenta were stained for human sFLT1 (1:200; AF231; R\&D Systems), as previously described (75). Slides were counterstained with hematoxylin, dehydrated, and mounted. Alternatively, tissues were embedded in OCT compound (Thermo Fisher) and snap-frozen in liquid nitrogen for immunofluorescence. Five-micrometer cryosections of thoracic aorta were cut and equilibrated in PBS for 10 minutes at $37^{\circ} \mathrm{C}$, followed by incubation with $5 \mathrm{nM}$ MitoSOX Red (Invitrogen) for 10 minutes at $37^{\circ} \mathrm{C}$. Slides were then rinsed with PBS, fixed, and mounted.
Statistics. Longitudinal data (hemodynamic data, serial sFLT1 measures) were compared using 2-way repeated-measures ANOVA, followed by Šidák's or Bonferroni's post test, as applicable. Data with multiple measurements per $n$ were compared using 2-way ANOVA. Data comparing 3 or more groups were analyzed using 1-way ANOVA with Tukey's multiple comparison test or Kruskal-Wallis with Dunn's post-test. Twogroup comparisons were made using paired or unpaired 2-tailed $t$ tests with or without Welch's correction, as applicable. Pearson $r$ test was used to assess correlation. GraphPad Prism version 6 was used for all analyses. A $P$ value of less than 0.05 was considered statistically significant.

Study approval. All animal procedures and surgical protocols were reviewed and approved by the IACUCs at Beth Israel Deaconess Medical Center and Tufts Medical Center. Beth Israel Deaconess Medical Center and Tufts Medical Center are accredited by the Association for Assessment and Accreditation of Laboratory Animal Care International. The IRB at Beth Israel Deaconess Medical Center approved collection and use of discarded human placentas.

\section{Author contributions}

SDB and SAK designed the study. SDB characterized the mouse model of preeclampsia including telemetry studies and performed rescue/safety experiments with sildenafil. ZKZ and IES performed all histology and immunohistochemistry studies. EVK and SDB performed ultrasound flow studies. ASL and AR conducted phosphorylation experiments. DZ measured renin and angiotensin. CDC, AW, DZ, and SDB performed PCR and immunoassays. JJD, AM, and MEM performed vascular reactivity studies. SDB, ZKZ, EVK, IES, IZJ, and SAK analyzed data. SDB and SAK wrote the manuscript with major contributions from EWS, PMK, IES, and IZJ. All authors reviewed the manuscript prior to submission.

\section{Acknowledgments}

S.D. Burke, C.D. Clark, A. Rajakumar, and S.A. Karumanchi were supported by the Howard Hughes Medical Institute. E.V. Khankin was supported by KO8 DK101568 from the NIH. I.Z. Jaffe was supported by RO1 grants HL095590 and HL119290 from the NIH.

Address correspondence to: Suzanne D. Burke, Beth Israel Deaconess Medical Center, 99 Brookline Avenue, RN-363, Boston, Massachusetts 02215, USA. Phone: 617.667.1514; E-mail: sdburke@bidmc.harvard.edu. Or to: S. Ananth Karumanchi, Beth Israel Deaconess Medical Center, 99 Brookline Avenue, RN-370D, Boston, Massachusetts 02215, USA. Phone: 617.667.1018; E-mail: sananth@bidmc.harvard.edu.
1. Powe CE, Levine RJ, Karumanchi SA. Preeclampsia, a disease of the maternal endothelium: the role of antiangiogenic factors and implications for later cardiovascular disease. Circulation. 2011;123(24):2856-2869.

2. Redman CW, Sargent IL. Latest advances in understanding preeclampsia. Science. 2005;308(5728):1592-1594.

3. Gant NF, Daley GL, Chand S, Whalley PJ, MacDonald PC. A study of angiotensin II pressor response throughout primigravid pregnancy. JClin Invest. 1973;52(11):2682-2689.

4. Chesley LC. Vascular reactivity in normal and toxemic pregnancy. Clin Obstet Gynecol. 1966;9(4):871-881.

5. Chesley LC, Talledo E, Bohler CS, Zuspan FP. Vascular reactivity to angiotensin ii and norepinephrine in pregnant women. Am JObstet Gynecol. 1965;91:837-842.

6. Dekker GA, Makovitz JW, Wallenburg HC. Prediction of pregnancy-induced hypertensive disorders by angiotensin II sensitivity and supine pressor test. Br JObstet Gynaecol. 1990;97(9):817-821.

7. Wallukat $\mathrm{G}$, et al. Patients with preeclampsia develop agonistic autoantibodies against the angiotensin AT1 receptor. J Clin Invest. 1999;103(7):945-952.

8. Wenzel K, et al. Angiotensin II type 1 receptor antibodies and increased angiotensin II sensitivity in pregnant rats. Hypertension. 2011;58(1):77-84.

9. AbdAlla S, Lother H, el Massiery A, Quitterer U. Increased AT1 receptor heterodimers in preeclampsia mediate enhanced angiotensin II responsiveness. Nat Med. 2001;7(9):1003-1009.

10. Quitterer U, Lother H, Abdalla S. AT1 receptor heterodimers and angiotensin II responsiveness in preeclampsia. Semin Nephrol. 2004;24(2):115-119.

11. Holobotovskyy V, et al. Regulator of $\mathrm{G}$ protein 
signaling 5 is a determinant of gestational hypertension and preeclampsia. Sci Transl Med. 2015;7(290):290ra88.

12. Alexander BT, Cockrell K, Cline FD, Llinas MT, Sedeek M, Granger JP. Effect of angiotensin II synthesis blockade on the hypertensive response to chronic reductions in uterine perfusion pressure in pregnant rats. Hypertension. 2001;38(3 pt 2):742-745.

13. Shah DM. Role of the renin-angiotensin system in the pathogenesis of preeclampsia. Am JPhysiol Renal Physiol. 2005;288(4):F614-F625.

14. Kendall RL, Thomas KA. Inhibition of vascular endothelial cell growth factor activity by an endogenously encoded soluble receptor. Proc Natl Acad Sci U S A. 1993;90(22):10705-10709.

15. Chaiworapongsa $\mathrm{T}$, et al. Plasma soluble vascular endothelial growth factor receptor-1 concentration is elevated prior to the clinical diagnosis of pre-eclampsia. J Matern Fetal Neonatal Med. 2005;17(1):3-18.

16. Chaiworapongsa T, et al. Evidence supporting a role for blockade of the vascular endothelial growth factor system in the pathophysiology of preeclampsia. Young Investigator Award. Am J Obstet Gynecol. 2004;190(6):1541-1547.

17. Levine RJ, et al. Circulating angiogenic factors and the risk of preeclampsia. $N$ EnglJMed. 2004;350(7):672-683.

18. Maynard SE, et al. Excess placental soluble fms-like tyrosine kinase 1 (sFlt1) may contribute to endothelial dysfunction, hypertension, and proteinuria in preeclampsia. JClin Invest. 2003;111(5):649-658.

19. Venditti CC, Casselman R, Young I, Karumanchi SA, Smith GN. Carbon monoxide prevents hypertension and proteinuria in an adenovirus sFlt-1 preeclampsia-like mouse model. PLoS One. 2014;9(9):e106502.

20. Bergmann A, et al. Reduction of circulating soluble Flt-1 alleviates preeclampsia-like symptoms in a mouse model. J Cell Mol Med. 2010;14(6B):1857-1867.

21. Seligman SP, Buyon JP, Clancy RM, Young BK, Abramson SB. The role of nitric oxide in the pathogenesis of preeclampsia. Am JObstet Gynecol. 1994;171(4):944-948.

22. Sandrim VC, Palei AC, Metzger IF, Gomes VA, Cavalli RC, Tanus-Santos JE. Nitric oxide formation is inversely related to serum levels of antiangiogenic factors soluble fms-like tyrosine kinase- 1 and soluble endogline in preeclampsia. Hypertension. 2008;52(2):402-407.

23. Sessa WC. eNOS at a glance. JCell Sci. 2004;117(pt 12):2427-2429.

24. Kroll J, Waltenberger J. VEGF-A induces expression of eNOS and iNOS in endothelial cells via VEGF receptor-2 (KDR). Biochem Biophys Res Commun. 1998;252(3):743-746.

25. Bussolati B, Dunk C, Grohman M, Kontos CD, Mason J, Ahmed A. Vascular endothelial growth factor receptor-1 modulates vascular endothelial growth factor-mediated angiogenesis via nitric oxide. Am J Pathol. 2001;159(3):993-1008.

26. Rafikov R, et al. eNOS activation and NO function: structural motifs responsible for the posttranslational control of endothelial nitric oxide synthase activity. JEndocrinol. 2011;210(3):271-284.
27. Kulandavelu S, Qu D, Adamson SL. Cardiovascular function in mice during normal pregnancy and in the absence of endothelial NO synthase. Hypertension. 2006;47(6):1175-1182.

28. Niu XF, Smith CW, Kubes P. Intracellular oxidative stress induced by nitric oxide synthesis inhibition increases endothelial cell adhesion to neutrophils. Circ Res. 1994;74(6):1133-1140.

29. Zhou XJ, Vaziri ND, Zhang J, Wang HW, Wang $\mathrm{XQ}$. Association of renal injury with nitric oxide deficiency in aged SHR: prevention by hypertension control with AT1 blockade. Kidney Int . 2002;62(3):914-921.

30. von Dadelszen P, Ornstein MP, Bull SB, Logan AG, Koren G, Magee LA. Fall in mean arterial pressure and fetal growth restriction in pregnancy hypertension: a meta-analysis. Lancet 2000;355(9198):87-92.

31. George EM, Palei AC, Dent EA, Granger JP. Sildenafil attenuates placental ischemia-induced hypertension. Am J Physiol Regul Integr Comp Physiol. 2013;305(4):R397-R403.

32. Herraiz S, et al. Sildenafil citrate improves perinatal outcome in fetuses from pre-eclamptic rats. BJOG. 2012;119(11):1394-1402.

33. Stanley JL, et al. Sildenafil citrate rescues fetal growth in the catechol-O-methyl transferase knockout mouse model. Hypertension. 2012;59(5):1021-1028.

34. Motta C, et al. Effect of sildenafil on pre-eclampsia-like mouse model induced By L-Name. Reprod Domest Anim. 2015;50(4):611-616.

35. March MI, et al. Circulating angiogenic factors and the risk of adverse outcomes among Haitian women with preeclampsia. PLoS One. 2015;10(5):e0126815.

36. Deng A, Engels K, Baylis C. Impact of nitric oxide deficiency on blood pressure and glomerular hemodynamic adaptations to pregnancy in the rat. Kidney Int. 1996;50(4):1132-1138.

37. Baylis C, Collins RC. Angiotensin II inhibition on blood pressure and renal hemodynamics in pregnant rats. Am J Physiol. 1986;250(2 pt 2):F308-F314

38. Baylis C. The determinants of renal hemodynamics in pregnancy. Am J Kidney Dis. 1987;9(4):260-264.

39. Robinson ES, et al. Suppression of the nitric oxide pathway in metastatic renal cell carcinoma patients receiving vascular endothelial growth factor-signaling inhibitors. Hypertension. 2010;56(6):1131-1136.

40. Gillis EE, Mooney JN, Garrett MR, Granger JP, Sasser JM. Sildenafil treatment ameliorates the maternal syndrome of preeclampsia and rescues fetal growth in the dahl salt-sensitive rat. Hypertension. 2016;67(3):647-653.

41. Bolnick JM, et al. Sildenafil stimulates human trophoblast invasion through nitric oxide and guanosine $3^{\prime}, 5^{\prime}$-cyclic monophosphate signaling. Fertil Steril. 2015;103(6):1587-1595.

42. Samangaya RA, et al. A randomised, doubleblinded, placebo-controlled study of the phosphodiesterase type 5 inhibitor sildenafil for the treatment of preeclampsia. Hypertens Pregnancy. 2009;28(4):369-382.

43. Krause BJ, Hanson MA, Casanello P. Role of nitric oxide in placental vascular development and function. Placenta. 2011;32(11):797-805.

44. Izumi H, Yallampalli C, Garfield RE. Gestational changes in L-arginine-induced relaxation of pregnant rat and human myometrial smooth muscle. Am JObstet Gynecol. 1993;169(5):1327-1337.

45. Yallampalli C, Garfield RE, Byam-Smith M. Nitric oxide inhibits uterine contractility during pregnancy but not during delivery. Endocrinology. 1993;133(4):1899-1902.

46. Lowe DT. Nitric oxide dysfunction in the pathophysiology of preeclampsia. Nitric Oxide. 2000;4(4):441-458.

47. Conrad KP, et al. Identification of increased nitric oxide biosynthesis during pregnancy in rats. FASEB J. 1993;7(6):566-571.

48. Fukumura D, et al. Predominant role of endothelial nitric oxide synthase in vascular endothelial growth factor-induced angiogenesis and vascular permeability. Proc Natl Acad Sci U S A. 2001;98(5):2604-2609.

49. Xue B, Singh M, Guo F, Hay M, Johnson AK. Protective actions of estrogen on angiotensin II-induced hypertension: role of central nitric oxide. Am J Physiol Heart Circ Physiol. 2009;297(5):H1638-H1646.

50. Li F, et al. eNOS deficiency acts through endothelin to aggravate sFlt-1-induced preeclampsia-like phenotype. J Am Soc Nephrol. 2012;23(4):652-660.

51. Ackermann A, Fernandez-Alfonso MS, Sanchez de Rojas R, Ortega T, Paul M, Gonzalez C. Modulation of angiotensin-converting enzyme by nitric oxide. Br J Pharmacol.1998;124(2):291-298.

52. Loscalzo J. Nitric oxide insufficiency, platelet activation, and arterial thrombosis. Circ Res. 2001;88(8):756-762.

53. Kulandavelu S, Whiteley KJ, Qu D, Mu J, Bainbridge SA, Adamson SL. Endothelial nitric oxide synthase deficiency reduces uterine blood flow, spiral artery elongation, and placental oxygenation in pregnant mice. Hypertension 2012;60(1):231-238.

54. Yallampalli C, Garfield RE. Inhibition of nitric oxide synthesis in rats during pregnancy produces signs similar to those of preeclampsia. Am J Obstet Gynecol. 1993;169(5):1316-1320.

55. Radi R. Nitric oxide, oxidants, and protein tyrosine nitration. Proc Natl Acad Sci U S A 2004;101(12):4003-4008.

56. Rajagopalan S, et al. Angiotensin II-mediated hypertension in the rat increases vascular superoxide production via membrane $\mathrm{NADH} /$ NADPH oxidase activation. Contribution to alterations of vasomotor tone. J Clin Invest. 1996;97(8):1916-1923.

57. Cai H, Harrison DG. Endothelial dysfunction in cardiovascular diseases: the role of oxidant stress. Circ Res. 2000;87(10):840-844.

58. Li L, Pan Y, Dai L, Liu B, Zhang D. Association of genetic polymorphisms on vascular endothelial growth factor and its receptor genes with susceptibility to coronary heart disease. Med Sci Monit. 2016;22:31-40.

59. Li W, Croce K, Steensma DP, McDermott DF, Ben-Yehuda O, Moslehi J. Vascular and metabolic implications of novel targeted cancer therapies: focus on kinase inhibitors. J Am Coll Cardiol. 2015;66(10):1160-1178.

60. Vigneau C, et al. All anti-vascular endothelial growth factor drugs can induce 'pre-eclampsia- 
like syndrome': a RARe study. Nephrol Dial Transplant. 2014;29(2):325-332.

61. Holwerda KM, et al. Hydrogen sulfide attenuates sFlt1-induced hypertension and renal damage by upregulating vascular endothelial growth factor. J Am Soc Nephrol. 2014;25(4):717-725.

62. Venkatesha S, et al. Soluble endoglin contributes to the pathogenesis of preeclampsia. Nat Med. 2006;12(6):642-649.

63. August $P$, et al. Longitudinal study of the reninangiotensin-aldosterone system in hypertensive pregnant women: deviations related to the development of superimposed preeclampsia. Am J Obstet Gynecol. 1990;163(5 pt 1):1612-1621.

64. Zhou CC, et al. Autoantibody from women with preeclampsia induces soluble Fms-like tyrosine kinase-1 production via angiotensin type 1 receptor and calcineurin/nuclear factor of activated T-cells signaling. Hypertension. 2008;51(4):1010-1019.

65. Irani RA, et al. The detrimental role of angioten- sin receptor agonistic autoantibodies in intrauterine growth restriction seen in preeclampsia. JExp Med. 2009;206(12):2809-2822.

66. Dechend R, et al. AT1 receptor agonistic antibodies from preeclamptic patients stimulate NADPH oxidase. Circulation. 2003;107(12):1632-1639.

67. Chen CW, Jaffe IZ, Karumanchi SA. Pre-eclampsia and cardiovascular disease. Cardiovasc Res. 2014;101(4):579-586.

68. Pruthi D, et al. Exposure to experimental preeclampsia in mice enhances the vascular response to future injury. Hypertension. 2015;65(4):863-870.

69. Mandala M, Osol G. Physiological remodelling of the maternal uterine circulation during pregnancy. Basic Clin Pharmacol Toxicol. 2012;110(1):12-18.

70. Saxena AR, Karumanchi SA, Brown NJ, Royle CM, McElrath TF, Seely EW. Increased sensitivity to angiotensin II is present postpartum in women with a history of hypertensive pregnancy. Hypertension. 2010;55(5):1239-1245.
71. Hladunewich MA, et al. Postpartum assessment of the renin angiotensin system in women with previous severe, early-onset preeclampsia. J Clin Endocrinol Metab. 2011;96(11):3517-3524.

72. Mongraw-Chaffin ML, Cirillo PM, Cohn BA. Preeclampsia and cardiovascular disease death: prospective evidence from the child health and development studies cohort. Hypertension. 2010;56(1):166-171.

73. Li Z, et al. Recombinant vascular endothelial growth factor 121 attenuates hypertension and improves kidney damage in a rat model of preeclampsia. Hypertension. 2007;50(4):686-692.

74. Burke SD, et al. Spiral arterial remodeling is not essential for normal blood pressure regulation in pregnant mice. Hypertension. 2010;55(3):729-737.

75. Rajakumar A, et al. Transcriptionally active syncytial aggregates in the maternal circulation may contribute to circulating soluble fms-like tyrosine kinase 1 in preeclampsia. Hypertension. 2012;59(2):256-264. 